The Annals of Probability

2002, Vol. 30, No. 3, 1003-1038

\title{
ON THE MINIMAL ENTROPY MARTINGALE MEASURE
}

\author{
By PETER GRANDITS ${ }^{1}$ AND ThORSTEN RHEINLÄNDER ${ }^{2}$ \\ University of Cambridge and Technische Universität Berlin
}

Let $X$ be a locally bounded semimartingale. Using the theory of $B M O$ martingales we give a sufficient criterion for a martingale measure for $X$ to minimize relative entropy among all martingale measures. This is applied to prove convergence of the $q$-optimal martingale measure to the minimal entropy martingale measure in entropy for $q \downarrow 1$ under the assumption that $X$ is continuous and that the density process of some equivalent martingale measure satisfies a reverse $L \log L$-inequality.

1. Introduction. The goal of this work is to study the measure $Q^{E}$ minimizing relative entropy $I(Q, P)$ with respect to a reference measure $P$ among all martingale measures. In particular we investigate its relationship to the family of $q$-optimal martingale measures, and relate properties of the density process of $Q^{E}$ to the closedness of certain spaces of stochastic integrals.

To be more precise, let us fix a locally bounded semimartingale $X$. We prove the following.

SUFFICIENT CRITERION. If there exists an equivalent martingale measure $\bar{Q}$ with finite relative entropy such that

(i) $\frac{d P}{d \bar{Q}} \in L^{\varepsilon}(P)$ for an $\varepsilon>0$,

(ii) $\frac{d \bar{Q}}{d P}=c \exp \left(\left(\int \eta d X\right)_{T}\right)$,

for a constant $c$ and an $X$-integrable $\eta$,

(iii) $\int \eta d X \in B M O(\bar{Q})$,

then $\bar{Q}$ is the minimal entropy martingale measure.

This criterion complements results given by Csiszár (1975) and recently in Frittelli (2000); its main advantage is that it involves only the "candidate measure" $\bar{Q}$.

$B M O$-techniques enter in a natural way into questions concerning relative entropy. This is due to the fact that a well known martingale inequality implies

Received October 2000; revised April 2001.

${ }^{1}$ Supported by the UBS.

${ }^{2}$ Supported by the DFG.

AMS 2001 subject classifications. 28D20, 60G48, 60H05, 91B28.

Key words and phrases. Relative entropy, martingale measures, $\mathrm{BMO}$-martingales. 
that the density process of a probability measure which is equivalent to $P$ and which has finite relative entropy is in the Hardy space $\mathcal{H}_{1}$, which is the dual space of $B M O$.

On the other hand, it was discovered by Delbaen et al. (1997) and by Grandits and Krawczyk (1998) that the theory of $B M O$-martingales is crucial in the study of the $q$-optimal martingale measure $Q^{(q)}$ [i.e., the measure with minimal $L^{q}(P)$-norm among all signed martingale measures] and its connection to the closedness in $L^{p}(P)$ of spaces of stochastic integrals with respect to $X$. Here and in the sequel we denote by $p$ and $q$ real numbers greater than 1 and such that $1 / p+1 / q=1$. To explain these results, define the space $G_{T}^{p}, p>1$, as the space of random variables $\left(\int \vartheta d X\right)_{T}$, where $T$ is some fixed time horizon, such that the stochastic integral process $\int \vartheta d X$ is in the space $\varsigma^{p}$ of semimartingales. If $X$ is in fact a local martingale, the Burkholder-Davis-Gundy (BDG) and the Doob inequalities already imply that $G_{T}^{p}$ is closed since it is isomorphic to a complete subspace of $L^{p}(P)$. In the semimartingale case, under the assumption that there exists a martingale measure for $X$, the question of closedness then boils down to whether weighted norm inequalities, that is, generalizations of the BDG and Doob inequalities can be derived. In fact, it turns out that it is therefore necessary that the density process of some martingale measure satisfies a reverse Hölder inequality; compare Doléans-Dade and Meyer (1979) or more recently Choulli, Krawczyk and Stricker $(1997,1998)$. It is well known that an exponential martingale $\&(M)$ satisfies a reverse Hölder inequality $R_{q}(P)$ for a $q>1$ if and only if both $M \in B M O(P)$ and there exists $h>0$ such that $\Delta M \geq-1+h$.

Let us now recall one of the main results in Delbaen et al. (1997) (in the case $p=2$ ) and Grandits and Krawczyk (1998) (in the case $p>1) . \mathcal{M}_{q}^{e}(P)$ denotes the space of equivalent martingale measures with $q$ th moments for the processes $\int \eta d X$ such that $\left(\int \eta d X\right)_{T} \in G_{T}^{p}$.

THEOREM. Let $X$ be continuous. Then the following assertions are equivalent:

(i) $\mathcal{M}_{q}^{e}(P) \neq \varnothing$ and $G_{T}^{p}$ is closed in $L^{p}(P)$;

(ii) the $q$-optimal martingale measure $Q^{(q)}$ is in $\mathcal{M}_{q}^{e}(P)$ and its density process $Z^{(q)}$ satisfies the reverse Hölder inequality $R_{q}(P)$.

This theorem is the starting point for us to draw useful conclusions from the above-mentioned sufficient criterion by restricting ourselves from now on to the case where $X$ is continuous. In particular we are interested in deriving the asymptotic relationship between $Q^{E}$ and the family of $q$-optimal martingale measures, provided that condition (i) in the closedness result above holds for some $q>1$. In concrete applications (e.g., in mathematical finance) it is often not difficult to show that this condition actually is satisfied.

It is useful for our purposes to introduce an analogue of the reverse Hölder inequality. 
Definition. A positive process $Z$ satisfies $R_{\operatorname{LLog} L}(P)$ if there exists $K>0$ such that

$$
\sup _{S}\left\|E\left[\frac{Z_{T}}{Z_{S}} \log ^{+} \frac{Z_{T}}{Z_{S}} \mid \mathcal{F}_{S}\right]\right\|_{\infty} \leq K .
$$

Here the supremum is taken over all stopping times $S \leq T$.

Using the theorem of Grandits and Krawczyk, we show that the $Z^{(q)}$ satisfy this $R_{\log L}(P)$-condition uniformly in $q$. The point is now, and this considerably simplifies our analysis, that it turns out that $R_{L \log L}(P)$ implies an $R_{p_{0}}(P)$ condition for a $p_{0}>1$. Therefore, BMO-theory essentially allows us to work in an $L^{p}$-framework while still covering a sufficiently general case. One might ask if it is possible to achieve full generality by using the Orlicz spaces $\log L(P)$ and $L_{\exp }(P)$. This, however, is not the case, as is shown by a simple counterexample at the end of Section 3. Let us now summarize the main results of Section 4.

It was proved in Grandits (1999) that for a bounded process $X$ in finite discrete time we have

$$
\lim _{q \rightarrow 1} \frac{d Q^{(q)}}{d P}=\frac{d Q^{E}}{d P} \quad \text { in } L^{1}(P) .
$$

The next result is a counterpart of this result for a continuous process $X$ in continuous time:

THEOREM. If there exists $Q \in \mathcal{M}_{q}^{e}(P)$ such that its density process satisfies $R_{L L o g L}(P)$, then

$$
\lim _{q \rightarrow 1} Q^{(q)}=Q^{E} \quad \text { in entropy. }
$$

It is well known that relative entropy does not induce a topology. We find it therefore remarkable that in our setting we can approximate a relative entropy minimizer by solutions to minimum norm problems.

Let us present the idea of the proof: If $X$ is continuous, it can be shown that the density of the $q$-optimal martingale measure has the following form:

$$
Z_{T}^{(q)}=C_{p}\left(1+\frac{f_{p}}{p-1}\right)^{p-1},
$$

where $C_{p}$ is a constant and $f_{p} \in K_{p}$; that is, $f_{p}$ is a stochastic integral with respect to $X$. Under suitable conditions, this should converge for $q \rightarrow 1$ (or $p \rightarrow \infty$ ) in some sense to

$$
\bar{Z}_{T}=c \exp (f),
$$

where $c=\lim _{p} C_{p}$ and $f$ is some limit of the $f_{p}$. To show this, we proceed as follows: we first show that the family $\left(Z_{T}^{(q)}\right)_{q}$ is uniformly integrable; hence we 
can extract weakly convergent subsequences. The main technical point is now to show that the convergence is even in $L^{1}(P)$. Up to now, we have not been able to do this in general. This is the point where the above-mentioned assumptions and techniques come into play: our strategy is to get better integrability properties by studying the density processes $Z^{(q)}$ rather than just the densities $Z_{T}^{(q)}$. As already explained, under some additional weak assumptions we prove that the $Z^{(q)}$ satisfy an $R_{p_{0}}(P)$-condition with a constant independent of $q$; hence the densities $Z_{T}^{(q)}$ are bounded in some $L^{\mu}(P)$-space for a $\mu>1$. Moreover, we show, again with the help of $B M O$-theory, that the $f_{p}$ are bounded in every $L^{r}(P)$-space. These two facts are essential for us to prove that we can extract from $\left(f_{p}\right)_{p}$ a subsequence which converges in probability. Having shown this, it is not hard to show that the $Z_{T}^{(q)}$ indeed converge in $L^{1}(P)$, at least along some subsequences, to the density $\bar{Z}_{T}$ (which may depend on the subsequence) of a martingale measure of the form in (1.1). Furthermore, we prove that $f$ from (1.1) is again a stochastic integral with respect to $X$, say

$$
f=\left(\int \eta d X\right)_{T}
$$

Since we also show that $\bar{Z}_{T}$ and $\int \eta d X$ meet the conditions of the sufficient criterion above, we finally can identify the limit measure with the minimal entropy martingale measure. As this measure is unique, we conclude that every subsequence converges to the same limit.

Finally, we provide a class of examples where one can calculate explicitly the minimal entropy martingale measure for a (in general) non-Markovian process $X$.

Let us remark that some of the methods developed in this paper have proven to be very useful in applications to mathematical finance. In particular, under the assumption that the density process of some martingale measure satisfies $R_{L \log L}(P)$, it was proved in Delbaen et al. (2000) that the problem of maximizing expected exponential utility from terminal wealth is dual to the problem of finding the minimal entropy martingale measure. The 2-optimal martingale measure on the other hand is crucial in the solution of the mean-variance hedging problem; see Rheinländer and Schweizer (1997). We hope that our main convergence result has shed some light on the relationship between these two problems, at least on the dual side of the corresponding optimal martingale measures.

2. Preliminaries. We begin by stating some general assumptions, which are valid throughout the whole text unless otherwise specified.

Let $(\Omega, \mathcal{F}, \mathbb{F}, P)$ be a filtered probability space, where the filtration $\mathbb{F}=$ $\left(\mathcal{F}_{t}\right)_{0 \leq t \leq T}$ satisfies the usual conditions and $\mathcal{F}_{0}$ is trivial. We denote by $X$ a locally bounded $\mathbb{R}^{d}$-valued semimartingale unless otherwise specified. Let $\mathcal{F}=\mathcal{F}_{T}$, where $T \in(0, \infty]$ is some time horizon. $C$ denotes a generic finite positive constant which may vary from line to line, and $S$ denotes a generic stopping time 
such that $0 \leq S \leq T$. With $p$ and $q$ we denote real numbers greater than 1 and such that $1 / p+1 / q=1$. For unexplained terminology we refer to Dellacherie and Meyer (1982).

Definition. $S_{\mathcal{V}}$ is the linear subspace of $L^{\infty}(\Omega, \mathcal{F}, P)$ spanned by the elementary stochastic integrals of the form $f=h^{\prime}\left(X_{T_{2}}-X_{T_{1}}\right)$, where $S \leq T_{1} \leq$ $T_{2} \leq T$ are stopping times such that the stopped process $X^{T_{2}}$ is bounded and $h$ is a bounded $\mathbb{R}^{d}$-valued $\widetilde{F}_{T_{1}}$-measurable random variable. Moreover, we set $\mathcal{V}={ }^{0} \mathcal{V}$.

DEFINITION. A signed martingale measure is a signed measure $Q \ll P$ with $E\left[\frac{d Q}{d P}\right]=1$ and $E\left[\frac{d Q}{d P} f\right]=0$ for all $f \in \mathcal{V}$.

We denote by $\mathcal{M}^{s}(P)$ the space of all signed martingale measures, by $\mathcal{M}(P)$ those elements of $\mathcal{M}^{s}(P)$ with nonnegative density and by $\mathcal{M}^{e}(P)$ the subset of $\mathcal{M}(P)$ consisting of probability measures which are equivalent to $P$. Subscripts refer to integrability properties of the densities, that is, $\mathcal{M}_{q}^{s}(P)=\mathcal{M}^{s}(P) \cap L^{q}(P)$, $\mathcal{M}_{q}^{e}(P)=\mathcal{M}^{e}(P) \cap L^{q}(P)$ and so on. Here and in the sequel we identify measures with their densities. Note that, as $X$ is locally bounded, a probability measure $Q$ absolutely continuous to $P$ is in $\mathcal{M}(P)$ if and only if $X$ is a local $Q$-martingale.

Elementary stochastic integrals are not sufficient for most applications in a continuous time framework. Therefore, one needs to define more general spaces of stochastic integrals. There are various concepts in the literature, and we shall now list some of them. The first one comes from Delbaen and Schachermayer (1996a).

Definition. The space ${ }^{S} K_{p}$ is the closure in $L^{p}(P)$ of ${ }^{S} \mathcal{V}$. We let $K_{p}={ }^{0} K_{p}$.

Let us now assume for the rest of this section that $X \in 8_{\text {loc }}^{p}$ (in particular $X$ is special), with canonical decomposition $X=X_{0}+M+A$, where $M$ is a local martingale with $M_{0}=0$ and $A$ is a predictable finite variation process. The next concept was introduced in Schweizer (1994). Here one considers those integrands $\vartheta$ for which the resulting stochastic integral process $\int \vartheta d X$ is in the space $\varsigma^{p}$ of semimartingales.

DEFINITION. Let $L^{p}(M)$ be the space of all $\mathbb{R}^{d}$-valued predictable processes $\vartheta$ such that

$$
\|\vartheta\|_{L^{p}(M)}:=E^{1 / p}\left[\left(\int \vartheta^{\prime} d[M] \vartheta\right)_{T}^{p / 2}\right]<\infty .
$$

Let $L^{p}(A)$ be the space of all $\mathbb{R}^{d}$-valued predictable processes $\vartheta$ such that

$$
\|\vartheta\|_{L^{p}(A)}:=E^{1 / p}\left[\left(\int\left|\vartheta^{\prime} d A\right|\right)_{T}^{p}\right]<\infty .
$$


We set $\Theta^{p}=L^{p}(M) \cap L^{p}(A)$ and

$$
G_{T}^{p}=\left\{\left(\int \vartheta d X\right)_{T} \mid \vartheta \in \Theta^{p}\right\}
$$

DEFINITION. For any RCLL process $Y$ we denote by $Y_{t}^{*}=\sup _{0 \leq s \leq t}\left|Y_{s}\right|$ the supremum process of $Y$. The space $\mathcal{R}^{p}(P)$ consists of all adapted RCLL processes $Y$ such that

$$
\|Y\|_{\mathcal{R}^{p}(P)}:=\left\|Y_{T}^{*}\right\|_{L^{p}(P)}<\infty .
$$

Where no confusion is possible, we use in the sequel, still denoted by the same symbols, the corresponding canonical quotient spaces modulo the zero-seminorm processes.

In Section 4 we use some results of Grandits and Krawczyk (1998) for a continuous semimartingale $X$. However, we still have to show that their notion of a signed martingale measure with $q$-integrable density coincides with our definition. They defined it in an analogous fashion as we did with the space $\mathcal{M}_{q}^{s}(P)$; however, they require their martingale measures to vanish on $G_{T}^{p}$ instead of $\mathcal{V}$. The equivalence of the two notions of martingale measures in the continuous case follows essentially from the results in Delbaen and Schachermayer (1996a), as is shown by the following.

LEMMA 2.1. If $X$ is continuous and $\mathcal{M}_{q}^{e}(P) \neq 0$, then $\overline{G_{T}^{p}}=K_{p}$, where the closure is taken in $L^{p}(P)$.

Proof. $\subset$. Due to the Burkholder-Davis-Gundy inequalities, we have, for $\vartheta \in \Theta^{p}$

$$
\begin{aligned}
\left\|\left(\int \vartheta d X\right)_{T}^{*}\right\|_{p} & \leq\left\|\left(\int \vartheta d M\right)_{T}^{*}\right\|_{p}+\left\|\left(\int \vartheta d A\right)_{T}^{*}\right\|_{p} \\
& \leq C\|\vartheta\|_{L^{p}(M)}+\|\vartheta\|_{L^{p}(A)} ;
\end{aligned}
$$

hence $\int \vartheta d X \in \mathcal{R}^{p}(P)$. Furthermore it follows from Doob's inequality that for any $Q \in \mathcal{M}_{q}^{e}(P)$ its density process $Z^{Q}$ is in $\mathcal{R}^{q}(P)$. Hence the local $P$-martingale $Z^{Q} \int \vartheta d X$ is in fact a uniformly integrable $P$-martingale, as its supremum process is integrable by Hölder's inequality. Since $X$ is continuous, the claim now follows by Theorems 1.2 and 2.2 of Delbaen and Schachermayer (1996a).

$\supset$. Let $Y_{T}=\left(\int \vartheta d X\right)_{T} \in \mathcal{V}$ and define $Y$ by $Y_{t}:=E_{Q}\left[Y_{T} \mid \mathcal{F}_{t}\right]$, where $E_{Q}$ denotes expectation with respect to a fixed $Q \in \mathcal{M}_{q}^{e}(P)$. $Y$ is uniformly bounded and, as $X$ is locally in $\varsigma^{p}, Y \in f_{\text {loc }}^{p}$ as well. Hence there exists a sequence of stopping times $T_{n} \uparrow T$ such that $\vartheta^{n}=\vartheta \mathbf{1}_{\left.] 0, T_{n}\right]} \in \Theta^{p}$ and consequently $Y_{T_{n}} \in G_{T}^{p}$. Furthermore,

$$
\lim _{n \rightarrow \infty} Y_{T_{n}}=Y_{T}, \quad Q \text { - and } P \text {-a.s., }
$$


and, since $Y$ is uniformly bounded, the convergence holds in $L^{p}(P)$ as well.

Let us now recall some definitions and results from $B M O$-theory.

NotATion. If we write $\sup _{S}$, it is always assumed that we take the essential supremum over all stopping times $S \leq T$.

In the following definitions we denote by $Q$ an arbitrary probability measure.

Definition. The Hardy space $\mathscr{H}_{1}(Q)$ consists of all $Q$-martingales $N$ such that

$$
\|N\|_{\mathscr{H}_{1}(Q)}=E_{Q}\left[N_{T}^{*}\right]<\infty
$$

Definition. $B M O(Q)$ is the space of all uniformly integrable $Q$-martingales $N$ vanishing at zero such that

$$
\|N\|_{B M O(Q)}=\sup _{S}\left\|E_{Q}\left[\left|N_{T}-N_{S-}\right| \mid \mathcal{F}_{S}\right]\right\|_{L^{\infty}(Q)}<\infty .
$$

Let us recall that $B M O(Q)$ is the dual space of $\mathscr{H}_{1}(Q)$.

In the sequel we denote by $Y$ a strictly positive adapted process.

Notation. For a stopping time $S \leq T$ we set

$$
{ }^{S} Y_{T}:=\frac{Y_{T}}{Y_{S}}
$$

Definition. $Z$ satisfies the reverse Hölder inequality $R_{p}(Q)$ if there exists $C$ such that

$$
\sup _{S} E_{Q}\left[\left.\left.\right|^{S} Y_{T}\right|^{p} \mid \mathcal{F}_{S}\right]<C .
$$

$Y$ satisfies the Muckenhoupt inequality $A_{p}(Q)$ if there exists $C$ such that

$$
\sup _{S} E_{Q}\left[\left.\left.\right|^{S} Y_{T}\right|^{-1 /(p-1)} \mid \mathcal{F}_{S}\right]<C .
$$

It turns out to be useful for our purposes to consider an analogue of the reverse Hölder inequality with the $L \log L$-function instead of a power function.

Definition. $\quad Y$ satisfies $R_{L \log L}(Q)$ with constant $K$ if there exists $K>0$ such that

$$
\sup _{S} E_{Q}\left[{ }^{S} Y_{T} \log { }^{+S^{S}} Y_{T} \mid \mathcal{F}_{S}\right]<K .
$$


REMARKS. Let $Z$ be the density process of a probability measure $Q \sim P$.

(i) It is straightforward to check that if $Z$ satisfies $R_{L \log L}(P)$ with constant $K$, then we have, for each stopping time $S \leq T$,

$$
E\left[Z_{T} \log Z_{T} \mid \mathcal{F}_{S}\right]<\left(K+\log Z_{S}\right) Z_{S} .
$$

Conversely, if (2.1) holds, then $Z$ satisfies $R_{L \log L}(P)$ with constant $K+\frac{1}{e}$ (since $\left.x \log ^{+} x \leq \frac{1}{e}+x \log x\right)$.

(ii) If $Z$ satisfies $R_{L L o g L}(P)$ with constant $K$, then for each stopping time $U \leq T$ the stopped process $Z^{U}$ satisfies $R_{L \log L}(P)$ with constant $K+\frac{1}{e}$. This follows from (i), since, as $Z \log Z$ is a $P$-submartingale, it follows that

$$
\begin{aligned}
E\left[Z_{T}^{U} \log Z_{T}^{U} \mid \mathcal{F}_{S}\right] & \leq E\left[E\left[Z_{T} \log Z_{T} \mid \mathcal{F}_{U}\right] \mid \mathcal{F}_{S}\right] \\
& =E\left[Z_{T} \log Z_{T} \mid \mathcal{F}_{S \wedge U}\right] \\
& \leq\left(K+\log Z_{S \wedge U}\right) Z_{S \wedge U} .
\end{aligned}
$$

Definition. $\quad Y$ satisfies condition $(S)$ if there exists $C$ such that

$$
\frac{1}{C} Y_{-} \leq Y \leq C Y_{-} .
$$

If especially $Y$ is of the form $Y=\mathscr{E}(N)$ for some semimartingale $N$ and $Y$ satisfies condition $(S)$, then we get, by an argument like that in the proof of Proposition 6 in Doléans-Dade and Meyer (1979), that there exist positive constants $j, J$ with $j \leq 1 / 2 \leq J$ such that

$$
\exp (N-J[N]) \leq \mathcal{E}(N) \leq \exp (N-j[N]) .
$$

Assume now that we have a probability measure $Q$ equivalent to $P$. We then set

$$
Z_{t}=E\left[\frac{d Q}{d P} \mid \mathscr{F}_{t}\right], \quad \widehat{Z}_{t}=E_{Q}\left[\frac{d P}{d Q} \mid \mathscr{F}_{t}\right] .
$$

Note that $Z \widehat{Z}=1$; hence $Z$ satisfies condition $(S)$ if and only if $\widehat{Z}$ satisfies condition $(S)$. Let

$$
M=\int \frac{d Z}{Z_{-}}, \quad \widehat{M}=\int \frac{d \widehat{Z}}{\widehat{Z}_{-}} ;
$$

therefore $Z=\mathcal{E}(M)$ and $\widehat{Z}=\mathscr{E}(\widehat{M})$. In this setting, we get the following.

LEMMA 2.2. If $Z$ satisfies $R_{L L o g L}(P)$ with constant $K_{L L o g L}$ as well as condition $(S)$ with constant s, then $M \in B M O(P)$ such that $\|M\|_{B M O_{2}} \leq J$ and $Z$ satisfies $R_{p}(P)$ for a $p>1$ with constant $K_{p}$. Here the finite constants $J$ and $K_{p}$ depend only on $K_{L L o g L}$ and $s$. 
PROOF. Our reasoning is close to the argument in the proof of Proposition 6 of Doléans-Dade and Meyer (1979). Let us assume first that $\widehat{M}$ is a uniformly integrable $Q$-martingale. Then we get from $(2.2), \mathcal{E}(M)=\mathscr{E}(\widehat{M})^{-1}$ and the Bayes formula

$$
\begin{aligned}
E_{Q}\left[[\widehat{M}]_{T}-[\widehat{M}]_{S} \mid \mathcal{F}_{S}\right] & =\frac{1}{j} E_{Q}\left[\widehat{M}_{S}-\widehat{M}_{T}+j\left([\widehat{M}]_{T}-[\widehat{M}]_{S}\right) \mid \mathcal{F}_{S}\right] \\
& \leq \frac{1}{j} E_{Q}\left[\log ^{+}\left({ }^{S} \mathcal{E}(\widehat{M})_{T}^{-1}\right) \mid \mathcal{F}_{S}\right] \\
& =\frac{1}{j} E\left[{ }^{S} Z_{T} \log ^{+S} Z_{T} \mid \mathcal{F}_{S}\right] .
\end{aligned}
$$

As $\widehat{Z}$ satisfies condition $(S)$, the jumps of $\widehat{M}$ are uniformly bounded. It therefore follows from $R_{\log L}(P)$ that $\widehat{M} \in B M O(Q)$. By localizing one can now easily conclude with the help of the preceding remark that this is true without assuming a priori that $\widehat{M}$ is a uniformly integrable $Q$-martingale. The statement about the constants now follows by a straightforward but tedious examination of the proofs in Doléans-Dade and Meyer (1979) or Kazamaki (1979); compare the Appendix for details.

3. On the minimal entropy martingale measure. In this section we assume that $X$ is a locally bounded $\mathbb{R}^{d}$-valued semimartingale.

Let us recall the concept of relative entropy which is also known as KullbackLeibler information. Our main references concerning relative entropy are Csiszár (1975) and Frittelli (2000).

DEFINITION. The relative entropy $I(Q, R)$ of the probability measure $Q$ with respect to the probability measure $R$ is defined as

$$
I(Q, R)= \begin{cases}E_{R}\left[\frac{d Q}{d R} \log \frac{d Q}{d R}\right], & \text { if } Q \ll R, \\ +\infty, & \text { otherwise. }\end{cases}
$$

It is well known that $I(Q, R) \geq 0$ and that $I(Q, R)=0$ if and only if $Q=R$.

DEFINITION. We say that a sequence $\left(Q_{n}\right)_{n}$ of probability measures converges in entropy to a probability measure $Q$ if

$$
\lim _{n \rightarrow \infty} I\left(Q_{n}, Q\right)=0 .
$$

DEFinition. The minimal entropy martingale measure $Q^{E}$ is the solution of

$$
\min _{Q \in \mathcal{M}(P)} I(Q, P) .
$$


Theorems 1, 2 and Remark 1 of Frittelli (2000) as well as the fact that $\mathcal{V} \subset$ $L^{\infty}(P)$ yield the following.

THEOREM 3.1. If there exists $Q \in \mathcal{M}^{e}(P)$ such that $I(Q, P)<\infty$, then the minimal entropy martingale measure exists, is unique and moreover is equivalent to $P$.

Let us now state a criterion for a martingale measure to coincide with the minimal entropy martingale measure. It is an easy consequence of results of Csiszár (1975) and Yor (1978). In the sequel, $\bar{Q}$ denotes a probability measure and $\bar{E}$ the expectation with respect to $\bar{Q}$.

Proposition 3.2. Assume there exists $\bar{Q} \in \mathcal{M}^{e}(P)$ with $I(\bar{Q}, P)<\infty$. Then $\bar{Q}=Q^{E}$ if and only if the following hold:

(i) $d \bar{Q} / d P=c \exp \left(\left(\int \eta d X\right)_{T}\right)$ for a constant c and an $X$-integrable $\eta$;

(ii) $E_{Q}\left[\left(\int \eta d X\right)_{T}\right]=0$ for $Q=\bar{Q}, Q^{E}$.

Proof. First note that, by Theorem $3.1, Q^{E}$ exists. To prove sufficiency, observe that we have, by (i),

$$
I(\bar{Q}, P)=\bar{E}\left[\log \frac{d \bar{Q}}{d P}\right]=\log c+\bar{E}\left[\left(\int \eta d X\right)_{T}\right]=\log c .
$$

On the other hand we get, by the positivity of the relative entropy as well as (i), (ii),

$$
\begin{aligned}
I\left(Q^{E}, P\right) & =I\left(Q^{E}, \bar{Q}\right)+E_{Q^{E}}\left[\log \frac{d \bar{Q}}{d P}\right] \\
& \geq \log c+E_{Q^{E}}\left[\left(\int \eta d X\right)_{T}\right] \\
& =\log c .
\end{aligned}
$$

It follows that

$$
I\left(Q^{E}, P\right) \geq I(\bar{Q}, P),
$$

and we conclude by the uniqueness of $Q^{E}$ that $\bar{Q}=Q^{E}$.

As regards necessity, it follows from Theorem 3.1 of Csiszár (1975) that $Q^{E}$ is of the form

$$
\frac{d Q^{E}}{d P}=c \exp f
$$

with $f \in \overline{\mathcal{V}}$ where the closure is taken in $L^{1}\left(Q^{E}\right)$. By the multidimensional version of a theorem of Yor [see Stricker (1990), Remark III.2] we conclude that $f=\left(\int \eta d X\right)_{T}$ for an $X$-integrable $\eta$. 
It turns out, however, that the sufficiency part of Proposition 3.2 is only of very limited use, since requirement (ii) usually is hard to verify, because it already involves $Q^{E}$. Hence it would be interesting to have a subset of all random variables $\left(\int \eta d X\right)_{T}$ such that (ii) holds which could be characterized more explicitly. In contrast to the criterion given recently in Frittelli [(2000), Theorems 3 and 4], the sufficient criterion we give in Proposition 3.4 has the advantage that it involves only the "candidate measure" $\bar{Q}$ and is thus verifiable in applications; compare the subsequent sections for examples.

Let us recall a useful martingale inequality [Revuz and Yor (1990), Remark after Theorem II.1.7] which implies that a right-continuous positive $\bar{Q}$-martingale $N$ with terminal value $N_{T} \in \operatorname{LLog} L(\bar{Q})$ is in $\mathscr{H}_{1}(\bar{Q})$, namely

$$
\bar{E}\left[N_{T}^{*}\right] \leq \frac{e}{e-1}\left(1+\bar{E}\left[N_{T} \log ^{+} N_{T}\right]\right) .
$$

Lemma 3.3. Assume there exists $\bar{Q} \in \mathcal{M}^{e}(P)$ with $I(\bar{Q}, P)<\infty$ such that

$$
\frac{d P}{d \bar{Q}} \in L^{\varepsilon}(P) \quad \text { for an } \varepsilon>0 .
$$

Then

$$
E_{Q}\left[\left(\int \eta d X\right)_{T}\right]=0
$$

for all $Q \in \mathcal{M}^{e}(P)$ with $I(Q, P)<\infty$ and all $\int \eta d X \in B M O(\bar{Q})$.

PROOF. Let $Q \in \mathcal{M}^{e}(P)$ with $I(Q, P)<\infty$ be arbitrary with density process $Z^{Q}$ and denote by $\bar{Z}$ the density process of our fixed martingale measure $\bar{Q}$. Then we have $Z^{Q}=\bar{Z} Y$ for a $\bar{Q}$-martingale $Y$ (it is the density process of $Q$ with respect to $\bar{Q})$. Since $\bar{Z}, Z^{Q}$ are positive, $Y$ is positive too and even in $\mathscr{H}_{1}(\bar{Q})$ :

$$
\begin{aligned}
\bar{E}\left[Y_{T}^{*}\right] & =\bar{E}\left[\left(Z^{Q} \frac{1}{\bar{Z}}\right)_{T}^{*}\right] \\
& \leq \frac{e}{e-1}\left(1+\bar{E}\left[Z_{T}^{Q} \frac{1}{\bar{Z}_{T}} \log ^{+}\left(Z_{T}^{Q} \frac{1}{\bar{Z}_{T}}\right)\right]\right) \\
& \leq \frac{e}{\varepsilon(e-1)}\left(\varepsilon+\varepsilon E\left[Z_{T}^{Q} \log ^{+} Z_{T}^{Q}\right]+E\left[Z_{T}^{Q} \log ^{+}\left(\frac{1}{\bar{Z}_{T}}\right)^{\varepsilon}\right]\right) \\
& \leq \frac{e}{\varepsilon(e-1)}\left(\varepsilon+(\varepsilon+1) E\left[Z_{T}^{Q} \log ^{+} Z_{T}^{Q}\right]+\frac{1}{e} E\left[\left(\frac{1}{\bar{Z}_{T}}\right)^{\varepsilon}\right]\right)<\infty,
\end{aligned}
$$


where we have used (3.1), (3.2) and the elementary inequality [see, e.g., Revuz and Yor (1990), Exercise II.1.16]

$$
a \log ^{+} b \leq a \log ^{+} a+\frac{1}{e} b \quad \text { for } a, b>0
$$

with $a=Z_{T}^{Q}$ and $b=\left(\frac{1}{\bar{Z}_{T}}\right)^{\varepsilon}$.

By Proposition 2 of Jeulin and Yor (1979) we have that if $U \in \mathscr{H}_{1}(\bar{Q})$ is positive and $V \in B M O(\bar{Q})$, then

$$
\bar{E}\left[U_{T} V_{T}\right]=\bar{E}\left[[U, V]_{T}\right]
$$

Hence

$$
E_{Q}\left[\left(\int \eta d X\right)_{T}\right]=\bar{E}\left[Y_{T}\left(\int \eta d X\right)_{T}\right]=\bar{E}\left[\left[Y, \int \eta d X\right]_{T}\right] .
$$

We are now going to show that $\left[Y, \int \eta d X\right]$ is a true $\bar{Q}$-martingale. It follows by integration by parts that

$$
\left[Y, \int \eta d X\right]=Y \int \eta d X-\int Y_{-} d\left(\int \eta d X\right)-\int\left(\int \eta d X\right)_{-} d Y .
$$

The last two summands on the right-hand side are local $\bar{Q}$-martingales since the integrands are locally bounded and the integrators are $\bar{Q}$-martingales. Concerning the first summand, note that $Y \int \eta d X$ is a local $\bar{Q}$-martingale if and only if $\int \eta d X$ is a local $Q$-martingale. $\left[\int \eta d X\right]$ is locally bounded, since it is the quadratic variation of a $B M O(\bar{Q})$-martingale, and the jumps of a $B M O$-martingale are uniformly bounded. Hence there exists a localizing sequence $\left(T_{n}\right)_{n}$ of stopping times such that, for all $n \in \mathbb{N}$,

$$
E_{Q}\left[\left(\int \eta^{\prime} d[X] \eta\right)_{T_{n}}^{1 / 2}\right]<\infty .
$$

By Proposition (2.48)(b) of Jacod (1979) it follows that $\int \eta d X$ is locally in $\mathscr{H}_{1}(Q)$. Summing up, we get that $\left[Y, \int \eta d X\right]$ is a local $\bar{Q}$-martingale. By Fefferman's inequality it is even a true $\bar{Q}$-martingale and we end up, by (3.3), with

$$
E_{Q}\left[\left(\int \eta d X\right)_{T}\right]=0
$$

This completes the proof.

From Proposition 3.2 and Lemma 3.3 we now get immediately the following sufficient criterion:

Proposition 3.4. Assume there exists $\bar{Q} \in \mathcal{M}^{e}(P)$ with $I(\bar{Q}, P)<\infty$ such that the following hold: 
(i) $\frac{d P}{d \bar{Q}} \in L^{\varepsilon}(P)$ for an $\varepsilon>0$;

(ii) $\frac{d \bar{Q}}{d P}=c \exp \left(\left(\int \eta d X\right)_{T}\right)$,

for a constant $c$ and an $X$-integrable $\eta$;

(iii) $\int \eta d X \in B M O(\bar{Q})$.

Then $\bar{Q}$ is the minimal entropy martingale measure.

Note in the context of the foregoing proposition that $f=\left(\int \eta d X\right)_{T} \in L_{\exp }(P)$, which follows readily from the John-Nirenberg and Hölder inequalities. However, neither is the criterion necessary nor is $f \in L_{\exp }(P)$ in general, as is shown by the following simple counterexample.

EXAMPLE. Let $B$ be a standard one-dimensional Brownian motion on $(\Omega, \mathcal{F}, P)$ and let $\mathbb{F}=\mathcal{F}^{B}$ be the augmented natural filtration of $B$. Consider the process $X$ given by

$$
X_{t}=B_{t}^{\tau}-t \wedge \tau, \quad 0 \leq t<\infty
$$

where

$$
\tau:=\inf _{t}\left\{B_{t}=1\right\}
$$

is a stopping time such that

$$
P(\tau<\infty)=1
$$

and

$$
E[\tau]=\infty
$$

In this situation we have one unique martingale measure $\widehat{P}$ for $X$ with finite relative entropy (and therefore it is the minimal entropy martingale measure), namely

$$
\frac{d \widehat{P}}{d P}=\exp \left(B_{\tau}-\frac{1}{2} \tau\right)=\exp \left(1-\frac{1}{2} \tau\right) .
$$

However, $d P / d \widehat{P} \notin L^{\varepsilon}(P)$ for any $\varepsilon>0$ :

$$
E\left[\exp \left(-\varepsilon+\frac{1}{2} \varepsilon \tau\right)\right] \geq \exp \left(-\varepsilon+\frac{1}{2} \varepsilon E[\tau]\right)=\infty .
$$

Moreover, if we write $\frac{d \widehat{P}}{d P}=c \exp (f)$, then $f \notin L_{\exp }(P)$. Otherwise, there would exist an $\varepsilon>0$ such that

$$
E\left[\left(\frac{d P}{d \widehat{P}}\right)^{\varepsilon}\right]=\frac{1}{c^{\varepsilon}} E\left[e^{-\varepsilon f}\right] \leq \frac{1}{c^{\varepsilon}} E\left[e^{\varepsilon|f|}\right]<\infty .
$$


4. The family of $q$-optimal martingale measures. In this section, we denote by $X$ a continuous $\mathbb{R}^{d}$-valued semimartingale with canonical decomposition $X=$ $X_{0}+M+A$. Here $M$ is a continuous local martingale and $A$ a finite variation process.

Let $\varphi_{p}:(-(p-1), \infty) \rightarrow(0, \infty)$ be the function given by

$$
x \mapsto\left(1+\frac{x}{p-1}\right)^{p-1}, \quad p>1 .
$$

It is well known that $\varphi_{p}(x)$ is increasing in $p$ to $\exp x$. Hence we have $\log \varphi_{p}(x) \leq x$.

DEFINITION. The q-optimal martingale measure $Q^{(q)}$ is the unique solution of

$$
\min _{Q \in \mathcal{M}_{q}^{s}(P)}\left\|\frac{d Q}{d P}\right\|_{L^{q}(P)} .
$$

Since $L^{p}$-spaces are uniformly convex, and $\mathcal{M}_{q}^{s}(P)$ is a closed affine subspace of $L^{q}(P)$, the $q$-optimal martingale measure always exists as long as $M_{q}^{s}(P)$ is nonempty.

Let us now recall some facts from optimization theory [compare Luenberger (1969)]. We say that $x \in L^{q}$ is aligned to $x^{*} \in L^{p}$ if $\left\langle x, x^{*}\right\rangle:=\left\|x x^{*}\right\|_{L^{1}}=$ $\|x\|_{L^{q}}\left\|x^{*}\right\|_{L^{p}}$ or equivalently if there exists $\gamma>0$ such that $x=\gamma \operatorname{sgn}\left(x^{*}\right)\left|x^{*}\right|^{p / q}$. Let $V$ denote some linear subspace of $L^{p}$. We set

$$
M=\left\{x \in L^{q} \mid\langle x, f\rangle=0 \forall f \in V ;\langle x, \mathbf{1}\rangle=0\right\}
$$

and

$$
A=\left\{x \in L^{q} \mid\langle x, f\rangle=0 \forall f \in V ;\langle x, \mathbf{1}\rangle=1\right\} .
$$

We assume that $A \neq \varnothing$. Since $L^{p}$-spaces are uniformly convex, there exists a unique solution to

$$
\min _{x \in A}\|x\|_{L^{q}} .
$$

In the next proof we use the following notation: Let $U \subset L^{q}, U^{*} \subset L^{p}$,

$$
\begin{aligned}
U^{\perp} & =\left\{x^{*} \in L^{p} \mid\left\langle u, x^{*}\right\rangle=0 \forall u \in U\right\}, \\
{ }^{\perp} U^{*} & =\left\{x \in L^{q} \mid\left\langle x, u^{*}\right\rangle=0 \forall u^{*} \in U^{*}\right\} .
\end{aligned}
$$

Moreover, we denote for $N \subset L^{p}$ with $\bar{N} w k$ (resp. $\bar{N} w k^{*}$ ) the closure of $N$ with respect to the weak (resp. weak ${ }^{*}$ ) topology.

A statement analogous to the following result can be found in Grandits (1999). 
LEMMA 4.1. $x_{0}$ is a solution of (4.2) if and only if $x_{0}$ is aligned to $c \cdot \mathbf{1}+f$, where $c \in \mathbb{R}$ and $f \in \bar{V}$. Here the closure is taken in $L^{p}$.

PROOF. Let $y \in A$. As $A=y-M$ it follows that $\min _{x \in A}\|x\|_{L^{q}}=$ $\min _{m \in M}\|y+m\|_{L^{q}}$. Let $x_{0}$ resp. $m_{0}$ denote the solutions, $x_{0}=y-m_{0}$. We get by Corollary 5.8.1 of Luenberger (1969) that $x_{0}$ is a solution of (4.2) if and only if it is aligned to an $x^{*} \in M^{\perp}$. Writing $N=\operatorname{span}(1+V)$, it remains to check that $M^{\perp}=\bar{N}$. This follows since $M={ }^{\perp} N$; hence $M^{\perp}=\left({ }^{\perp} N\right)^{\perp}=\bar{N} w k^{*}$. As $L^{p}$-spaces are reflexive, $\bar{N} w k^{*}=\bar{N} w k$ and, as $N$ is a subspace, hence convex, $\bar{N} w k=\bar{N}$.

From the preceding lemma, we immediately get the following result.

Proposition 4.2. The q-optimal martingale measure $Q^{(q)}$ is aligned to $1+f_{p} /(p-1)$ for an $f_{p} \in K_{p}=\overline{\mathcal{V}}$, where the closure is taken in $L^{p}(P)$. That is, there exists a constant $C_{p}$ such that

$$
\frac{d Q^{(q)}}{d P}=C_{p} \operatorname{sgn}\left(1+\frac{f_{p}}{p-1}\right)\left|1+\frac{f_{p}}{p-1}\right|^{p-1} .
$$

Conversely, if there exists an element of $\mathcal{M}_{q}^{s}(P)$ which is of the form (4.3), then it is the q-optimal martingale measure.

A priori, the $q$-optimal martingale measure is a signed measure. In our case ( $X$ is continuous), however, we have the following result which was mentioned in Grandits and Krawczyk [(1998), proof of Lemma 4.1]; compare also Theorem 3.1 of Delbaen and Schachermayer (1996b), where it is proved for $p=2$.

Proposition 4.3. If $X$ is continuous and $\mathcal{M}_{q}^{e}(P) \neq \varnothing$, then $Q^{(q)}$ is equivalent to $P$.

This fundamental result will be used throughout the whole text without further reference. It is crucial for the proofs of our results to consider not only the densities of the $q$-optimal martingale measures but also their density processes $Z^{(q)}$. Let us therefore recall some definitions and results of Krawczyk (1997).

DEFINITION. Let $S \leq T$ be a stopping time. A random variable $Z \in L^{1}(P)$ is an $\mathcal{F}_{S}$-normalized martingale measure if the following hold:

(i) $E\left[Z f \mid \mathcal{F}_{S}\right]=0$ for all $f \in{ }^{S} \mathcal{V}$;

(ii) $E\left[Z \mid \mathcal{F}_{S}\right]=1$.

Note that our definition differs slightly from that of Krawczyk (1997) since we do not require that

$$
E\left[|Z|^{q} \mid \mathcal{F}_{S}\right]<\infty .
$$


REMARK. Every equivalent martingale measure $Z_{T}$ induces by Bayes's formula the family of $\mathcal{F}_{S}$-normalized martingale measures $\left({ }^{S} Z_{T}\right)$, where $S$ runs through all stopping times $S \leq T$.

Whereas Krawczyk (1997) considers an arbitrary linear subspace $G$ of $L^{p}(P)$, we shall state his results only in the special case $G={ }^{S} \mathcal{V}$. To apply his results, let us notice furthermore that the following hold:

(i) ${ }^{S} \mathcal{V}$ is $\mathcal{F}_{S}$-invariant; that is, for any $A \in \mathcal{F}_{S}$ and each $f \in{ }^{S} \mathcal{V}$, we have that $\mathbf{1}_{A} f \in{ }^{S} \mathcal{V}$

(ii) if $\mathcal{M}_{q}^{e} \neq \varnothing$, then ${ }^{S} \mathcal{V}$ satisfies $\mathcal{F}_{S}-Q N A$ ("quasi-no-arbitrage"); that is, $A \in \mathcal{F}_{S}$ and $\mathbf{1}_{A} \in{ }^{S} K_{p}$ imply that $P(A)=0$.

While (i) is obvious from the definition of ${ }^{S} \mathcal{V}$, (ii) follows immediately from the fact that ${ }^{S} K_{p} \subset K_{p}$ : let $Q \in \mathcal{M}_{q}^{e}$; then $Q(A)=0$ if $\mathbf{1}_{A} \in{ }^{S} K_{p}$, and, since $Q$ is equivalent to $P$, this implies that $P(A)=0$. Now let $-{ }^{S} \widetilde{f}_{p}$ be the projection of 1 in $L^{p}(P)$ onto ${ }^{S} K_{p}$ and let

$$
S_{g_{p}}=\left|1+{ }^{S} \widetilde{f}_{p}\right|^{p-1} .
$$

Note that in Krawczyk's notation, our $-{ }^{S} \widetilde{f}_{p}$ corresponds to his $g$ and our ${ }^{S} g_{p}$ to his $f$. By Krawczyk [(1997), Proposition 4.4], we get, as ${ }^{S} \mathcal{V}$ satisfies $\mathcal{F}_{S}$-QNA,

$$
E\left[{ }^{S} g_{p} \mid \mathcal{F}_{S}\right] \neq 0, \quad P \text {-a.s.; }
$$

hence we may put

$$
S_{\mathfrak{Z}^{(q)}}:=\frac{S_{g_{p}}}{E\left[{ }^{S} g_{p} \mid \mathcal{F}_{S}\right]} .
$$

Now the following proposition follows immediately from Krawczyk (1997), Proposition 4.7.

Proposition 4.4. Let $\mathcal{M}_{q}^{e} \neq \varnothing$. For each stopping time $S \leq T$ we have that the following hold:

(i) ${ }^{S} \mathfrak{Z}^{(q)}$ is the density of an $\mathcal{F}_{S}$-normalized martingale measure;

(ii) $E\left[\left.\left.\right|^{S} \mathfrak{Z}^{(q)}\right|^{q} \mid \mathcal{F}_{S}\right]<\infty$;

(iii) if $Z$ is the density of an $\mathcal{F}_{S}$-normalized martingale measure, then

$$
E\left[\left|{ }^{S} \mathfrak{Z}^{(q)}\right|^{q} \mid \mathcal{F}_{S}\right] \leq E\left[|Z|^{q} \mid \mathcal{F}_{S}\right] ;
$$

(iv) there exists an $\mathcal{F}_{S}$-measurable $C_{p, S}$ such that

$$
S_{\mathfrak{Z}^{(q)}}=C_{p, S}\left|1+{ }^{S} \tilde{f}_{p}\right|^{p-1} ;
$$

(v) $S \tilde{f}_{p}$ solves the minimization problem

$$
\min _{f \in{ }^{S} K_{p}} E\left[|1+f|^{p} \mid \mathcal{F}_{S}\right] .
$$


PROOF. It only remains to show (v). We have, by (i),

$$
E\left[{ }^{S} \mathfrak{Z}^{(q)}(1+f) \mid \mathcal{F}_{S}\right]=1 \quad \text { for all } f \in{ }^{S} K_{p} .
$$

The conditional Hölder inequality yields

$$
E^{-1 / q}\left[\left|{ }^{S} \mathfrak{Z}^{(q)}\right|^{q} \mid \mathcal{F}_{S}\right] \leq E^{1 / p}\left[|1+f|^{p} \mid \mathcal{F}_{S}\right] .
$$

By formula (4.4) of Krawczyk (1997) we get

$$
E^{1 / p}\left[\left|1+{ }^{S} \tilde{f}_{p}\right|^{p} \mid \mathcal{F}_{S}\right]=E^{-1 / q}\left[\left.\left.\right|^{S} \mathfrak{Z}^{(q)}\right|^{q} \mid \mathcal{F}_{S}\right] .
$$

This completes the proof.

REMARK. By the uniqueness of $Q^{(q)}$, it follows easily that ${ }^{S} \mathfrak{Z}^{(q)} Z_{S}^{(q)}=Z_{T}^{(q)}$, provided that $\mathcal{M}_{q}^{e}(P) \neq \varnothing$; hence ${ }^{S} Z_{T}^{(q)}$ is a version of ${ }^{S} \mathfrak{Z}^{(q)}$.

Let us now state the main result about the closedness of the spaces $G_{T}^{p}$ in $L^{p}(P)$, which was obtained by Grandits and Krawczyk (1998).

THEOREM 4.5. Let $X$ be continuous. Then the following are equivalent:

(i) $\mathcal{M}_{q}^{e}(P) \neq \varnothing$ and $G_{T}^{p}$ is closed in $L^{p}(P)$;

(ii) the density process of some $Q \in \mathcal{M}_{q}^{e}(P)$ satisfies $R_{q}(P)$;

(iii) the q-optimal martingale measure $Q^{(q)}$ is in $\mathcal{M}_{q}^{e}(P)$ and its density process $Z^{(q)}$ satisfies $R_{q}(P)$.

Let us formulate another equivalent assertion involving the $R_{L L o g L}$-condition, after the following preparatory result.

LEMMA 4.6. If the density process $Z^{E}$ of the minimal entropy martingale measure $Q^{E}$ satisfies $R_{L \log L}(P)$, then it also satisfies condition $(S)$.

PROOF. It follows from Proposition 3.2 that

$$
Z_{T}^{E}=c \exp \left(\left(\int \eta d X\right)_{T}\right)
$$

where $\left(\int \eta d X\right)_{T}$ is in the $L^{1}\left(Q^{E}\right)$-closure of $\mathcal{V}$. Therefore we get by Remark III.2 of Stricker (1990) that

$$
\begin{aligned}
\widehat{Z}_{t} & :=\exp E_{Q^{E}}\left[\log Z_{T}^{E} \mid \mathcal{F}_{t}\right] \\
& =c \exp \left(\left(\int \eta d X\right)_{t}\right) .
\end{aligned}
$$


As $X$ is continuous, it follows that $\widehat{Z}$ is continuous too. By Jensen's inequality and the Bayes formula,

$$
\begin{aligned}
Z_{S}^{E} \log Z_{S}^{E} & =E\left[Z_{T}^{E} \mid \mathcal{F}_{S}\right] \log \left(E\left[Z_{T}^{E} \mid \mathcal{F}_{S}\right]\right) \\
& \leq E\left[Z_{T}^{E} \log Z_{T}^{E} \mid \mathcal{F}_{S}\right] \\
& =Z_{S}^{E} E_{Q^{E}}\left[\log Z_{T}^{E} \mid \mathcal{F}_{S}\right] \\
& =Z_{S}^{E} \log \widehat{Z}_{S}
\end{aligned}
$$

hence

$$
Z_{S}^{E} \leq \widehat{Z}_{S}
$$

On the other hand, as $x \log x \leq x \log ^{+} x, R_{L \log L}(P)$ implies that there exists $C$ such that

$$
E\left[\frac{Z_{T}^{E}}{Z_{S}^{E}}\left(\log Z_{T}^{E}-\log Z_{S}^{E}\right) \mid \mathcal{F}_{S}\right] \leq C,
$$

and thus we get

$$
\begin{aligned}
Z_{S}^{E} \log \widehat{Z}_{S} & =E\left[Z_{T}^{E} \log Z_{T}^{E} \mid \mathcal{F}_{S}\right] \\
& \leq C Z_{S}^{E}+E\left[Z_{T}^{E} \log Z_{S}^{E} \mid \mathcal{F}_{S}\right] \\
& =Z_{S}^{E}\left(C+\log Z_{S}^{E}\right),
\end{aligned}
$$

and therefore

$$
\widehat{Z}_{S} \leq e^{C} Z_{S}^{E}
$$

As $\widehat{Z}$ is continuous, (4.4) together with (4.5) implies that $Z^{E}$ satisfies condition $(S)$.

Now we can formulate the following.

PROPOSITION 4.7. The following assertions are equivalent ( $X$ is assumed to be continuous):

(i) there exists $q>1$ such that $\mathcal{M}_{q}^{e}(P) \neq \varnothing$ and $G_{T}^{p}$ is closed in $L^{p}(P)$;

(ii) the density process $Z^{Q}$ of some $Q \in \mathcal{M}_{q}^{e}(P)$ satisfies $R_{\log L}(P)$;

(iii) the density process $Z^{E}$ of the minimal entropy martingale measure satisfies $R_{L \log L}(P)$.

ProOF. (i) $\Rightarrow$ (ii). This follows by Theorem 4.1 of Grandits and Krawczyk (1998) and the fact that $R_{q}(P)$ implies $R_{L L o g L}(P)$.

(ii) $\Rightarrow$ (iii). This is a consequence of Lemma 4 of Delbaen et al. (2000). 
(iii) $\Rightarrow$ (i). Since by the preceding lemma $R_{L \log L}(P)$ implies that $Z^{E}$ satisfies condition $(S)$, by Lemma 2.2 it also satisfies $R_{q}(P)$ for a $q>1$. Hence the statement follows again by Theorem 4.1 of Grandits and Krawczyk (1998).

Let us now impose the following.

STANDING ASSUMPTIONS. For the continuous semimartingale $X$, there exists some $Q \in \mathcal{M}_{q_{0}}^{e}(P)$ for a $q_{0}>1$, and the density process of $Q$ satisfies $R_{L L o g L}(P)$.

These assumptions are sufficient to derive the $R_{L \log L}(P)$-inequality uniformly for the whole family of $q$-optimal martingale measures.

LEMMA 4.8. Under the standing assumptions,

$$
\sup _{q \leq q_{0}} \sup _{S} E\left[{ }^{S} Z_{T}^{(q)} \log { }^{+S} Z_{T}^{(q)} \mid \mathcal{F}_{S}\right]<C .
$$

ProOF. Recall the formula for ${ }^{S} Z_{T}^{(q)}$ in Proposition 4.4 and let ${ }^{S} f_{p}:=$ $(p-1)^{S} \widetilde{f}_{p}$. Thus

$$
{ }^{S} Z_{T}^{(q)}=C_{p, S}\left(1+\frac{{ }^{S} f_{p}}{p-1}\right)^{p-1}=C_{p, S} \varphi_{p}\left({ }^{S} f_{p}\right)>0 .
$$

Since $x \log ^{+} x \leq 1 / e+x \log x$ for $x>0$ and $\log \varphi_{p}(x) \leq x$ for $x>-(p-1)$, we get, together with the fact that ${ }^{S} f_{p}>-(p-1)$ since $Z_{T}^{(q)}>0$,

$$
\begin{aligned}
\sup _{p \geq p_{0}} \sup _{S} E\left[\varphi_{p}\left({ }^{S} f_{p}\right) \log ^{+} \varphi_{p}\left({ }^{S} f_{p}\right) \mid \mathcal{F}_{S}\right] \\
\leq \frac{1}{e}+\sup _{p \geq p_{0}} \sup _{S} E\left[\varphi_{p}\left({ }^{S} f_{p}\right) \log \varphi_{p}\left({ }^{S} f_{p}\right) \mid \mathcal{F}_{S}\right] \\
\leq \frac{1}{e}+\sup _{p \geq p_{0}} \sup _{S} E\left[\varphi_{p}\left({ }^{S} f_{p}\right)^{S} f_{p} \mid \mathcal{F}_{S}\right] \\
=\frac{1}{e}+\sup _{p \geq p_{0}} \sup _{S} \frac{1}{C_{p, S}} E\left[{ }^{S} Z_{T}^{(q)}{ }^{S} f_{p} \mid \mathcal{F}_{S}\right] \\
=\frac{1}{e} .
\end{aligned}
$$

It remains to show that

$$
\sup _{q \leq q_{0}} \sup _{S} C_{p, S} \leq C
$$


Since ${ }^{S} Z_{T}^{(q)}$ is the density of an $\mathcal{F}_{S}$-normalized martingale measure and $p-1=$ $p / q, q-1=q / p$, we get

$$
\begin{aligned}
E\left[\left({ }^{S} Z_{T}^{(q)}\right)^{q} \mid \mathcal{F}_{S}\right] & =\left(C_{p, S}\right)^{q} E\left[\left(1+\frac{{ }^{S} f_{p}}{p-1}\right)^{p} \mid \mathcal{F}_{S}\right] \\
& =\left(C_{p, S}\right)^{q-1} E\left[C_{p, S}\left(1+\frac{{ }^{S} f_{p}}{p-1}\right)^{p-1}\left(1+\frac{{ }^{S} f_{p}}{p-1}\right) \mid \mathcal{F}_{S}\right] \\
& =\left(C_{p, S}\right)^{q-1} E\left[S_{Z_{T}^{(q)}}\left(1+\frac{{ }^{S} f_{p}}{p-1}\right) \mid \mathcal{F}_{S}\right] \\
& =\left(C_{p, S}\right)^{q / p} .
\end{aligned}
$$

Hence we have the representation

$$
C_{p, S}=E^{p / q}\left[\left({ }^{S} Z_{T}^{(q)}\right)^{q} \mid \mathcal{F}_{S}\right] .
$$

Let

$$
I\left(q_{0}\right)=\left\{q \mid\left[p_{0}-1\right] \leq p \leq\left[p_{0}-1\right]+1\right\} .
$$

Here $[x]$ denotes the integral part of $x$.

By Proposition 4.4(iii) and by the conditional Jensen inequality as well as the reverse Hölder inequality $R_{q_{0}}(P)$ we get, for $q \leq q_{0}$,

$$
\begin{aligned}
E^{1 / q}\left[\left({ }^{S} Z_{T}^{(q)}\right)^{q} \mid \mathcal{F}_{S}\right] & \leq E^{1 / q}\left[\left({ }^{S} Z_{T}^{\left(q_{0}\right)}\right)^{q} \mid \mathcal{F}_{S}\right] \\
& \leq E^{1 / q_{0}}\left[\left({ }^{S} Z_{T}^{\left(q_{0}\right)}\right)^{q_{0}} \mid \mathcal{F}_{S}\right] \\
& <C,
\end{aligned}
$$

where $C$ is independent of $q$ and $S$. Combining this with (4.7), it follows that

$$
\sup _{q \in I\left(q_{0}\right)} \sup _{S} C_{p, S}<C .
$$

Now let $q_{2}<q_{1}$ be such that $p_{2}=p_{1}+1$, where $1 / p_{i}+1 / q_{i}=1$ for $i=1,2$. By Proposition 4.4(i) and (v), we get

$$
\begin{aligned}
\left(C_{p_{1}, S}\right)^{-1} & =E\left[\left(1+\frac{{ }^{S} f_{p_{1}}}{p_{1}-1}\right)^{p_{1}-1} \mid \mathcal{F}_{S}\right] \\
& =E\left[\left(1+\frac{{ }^{S} f_{p_{1}}}{p_{1}-1}\right)^{p_{1}} \mid \mathcal{F}_{S}\right] \\
& \leq E\left[\left(1+\frac{{ }^{S} f_{p_{2}}}{p_{2}-1}\right)^{p_{1}} \mid \mathcal{F}_{S}\right] \\
& =\left(C_{p_{2}, S}\right)^{-1}
\end{aligned}
$$


hence

$$
C_{p_{2}, S} \leq C_{p_{1}, S}
$$

Therefore our claim (4.6) is proved.

COROLLARY 4.9. Under our standing assumptions, for every sequence $\left(q_{n}\right)_{n}$ with $q_{n} \downarrow 1$ we can extract a subsequence, still denoted by $\left(q_{n}\right)_{n}$, such that there exists a signed martingale measure with density $\bar{Z}_{T}$ (which a priori may depend on the chosen sequence) with

$$
\lim _{q_{n} \rightarrow 1} Z_{T}^{\left(q_{n}\right)}=\bar{Z}_{T} \quad \text { weakly in } L^{1}(P) .
$$

Proof. As the family $\left(Z_{T}^{(q)}\right)_{q>1}$ is uniformly integrable by the preceding lemma, the statement follows from the Dunford-Pettis compactness criterion.

REMARK. We do not need the reverse Hölder inequality in order to derive this corollary. To see this, it suffices to go through the proof of Lemma 4.8 in the special case where $S=0$.

LEMMA 4.10. Under the standing assumptions, the $Z^{(q)}$ satisfy condition $(S)$ uniformly in $q$.

PROOF. It was proved in Grandits and Krawczyk [(1998), Lemma 4.2] that the density process $Z^{(q)}$ of $Q^{(q)}, q \leq q_{0}$, satisfies condition $(S)$ with constant $\left(R_{q}\right)^{p / q}$, where

$$
R_{q}:=\sup _{S}\left\|E\left[\left({ }^{S} Z_{T}^{(q)}\right)^{q} \mid \mathcal{F}_{S}\right]\right\|_{L^{\infty}(P)} .
$$

It follows by (4.7) and (4.6) that

$$
\sup _{q \leq q_{0}}\left(R_{q}\right)^{p / q}=\sup _{q \leq q_{0}} \sup _{S}{ }^{S} C_{p} \leq C ;
$$

hence the statement is proved.

Next we need to derive bounds on the ${ }^{S} f_{p}$.

LEMMA 4.11. Under our standing assumptions we have

$$
\sup _{p \geq r+1} \sup _{S} E\left[\left({ }^{S} f_{p}^{+}\right)^{r} \mid \mathcal{F}_{S}\right]<C \equiv C(r) \quad \text { for each } r \geq p_{0} .
$$


Proof. As $\varphi_{p}(x)$ is increasing in $p$ for $x>0$, we get $\left(p-1 \geq r \geq p_{0}\right)$

$$
\begin{aligned}
r^{-r} E\left[\left({ }^{S} f_{p}^{+}\right)^{r} \mid \mathcal{F}_{S}\right] & \leq E\left[\mathbf{1}_{\left({ }^{S} f_{p}>0\right)}\left(1+\frac{{ }^{S} f_{p}}{r}\right)^{r} \mid \mathcal{F}_{S}\right] \\
& \leq E\left[\mathbf{1}_{\left(S_{\left.f_{p}>0\right)}\left(1+\frac{{ }^{S} f_{p}}{p-1}\right)^{p-1} \mid \mathcal{F}_{S}\right]}\right. \\
& \leq E\left[\left(1+\frac{{ }^{S} f_{p}}{p-1}\right)^{p-1} \mid \mathcal{F}_{S}\right] \\
& =E\left[\left(1+\frac{S f_{p}}{p-1}\right)^{p} \mid \mathcal{F}_{S}\right] \\
& \leq 1,
\end{aligned}
$$

where the third inequality follows from the positivity of the ${ }^{S} Z_{T}^{(q)}$ and the last two lines follow from Proposition 4.4 and the fact that $0 \in{ }^{S} \mathcal{V}$. Note that these last two lines can be written alternatively as

$$
\left(C_{p, S}\right)^{-1} \leq 1
$$

This completes the proof.

While the $L^{r}$-bounds on the positive parts of the ${ }^{S} f_{p}$ have been derived by elementary arguments, we need arguments from $B M O$-theory in obtaining bounds for the negative parts.

LEMMA 4.12. Under our standing assumptions, there exists $\alpha>0$, independent of $p$, such that

$$
\sup _{p \geq p_{0}} \sup _{S} E\left[\exp \left(\alpha^{S} f_{p}^{-}\right) \mid \mathcal{F}_{S}\right]<C
$$

PROOF. Since $\varphi_{p}(x) \leq \exp (x)$ for $x>-(p-1)$ and

$$
\varphi_{p}\left({ }^{S} f_{p}\right)=\left(C_{p, S}\right)^{-1 S} Z_{T}^{(q)},
$$

we have

$$
{ }^{S} f_{p}^{-} \leq\left|\log \left(\left(C_{p, S}\right)^{-1 S} Z_{T}^{(q)}\right)\right| .
$$

As $1 \leq C_{p, S} \leq C$ by (4.8) and (4.6), we get

$$
{ }^{S} f_{p}^{-} \leq \log C+\left|\log \left({ }^{S} Z_{T}^{(q)}\right)\right| .
$$

Since $Z^{(q)}>0$ there exists a local $P$-martingale $M^{(q)}$ such that $Z^{(q)}=\mathcal{E}\left(M^{(q)}\right)$. By the proof of Lemma 4.10, the $Z^{(q)}$ satisfy condition $(S)$ uniformly in $q$. Therefore we get by (2.2) and the John-Nirenberg inequalities [cf. Kazamaki 
(1994), Theorems 2.1,2.2, where they are stated in the continuous case together with his remarks that they are also true in general] that

$$
\begin{aligned}
\sup _{S} E & {\left[\exp \left(\alpha\left|\log \left({ }^{S} Z_{T}^{(q)}\right)\right|\right) \mid \mathcal{F}_{S}\right] } \\
\leq & \sup _{S} E\left[\exp \left(\alpha\left|M_{T}^{(q)}-M_{S}^{(q)}-j\left(\left[M^{(q)}\right]_{T}-\left[M^{(q)}\right]_{S}\right)\right|\right) \mid \mathcal{F}_{S}\right] \\
\leq & \sup _{S} E^{1 / 2}\left[\exp \left(2 \alpha\left|M_{T}^{(q)}-M_{S}^{(q)}\right|\right) \mid \mathcal{F}_{S}\right] \\
& \times E^{1 / 2}\left[\exp \left(2 \alpha j\left(\left[M^{(q)}\right]_{T}-\left[M^{(q)}\right]_{S}\right)\right) \mid \mathcal{F}_{S}\right] \\
\leq & 2
\end{aligned}
$$

provided that

$$
\alpha \leq \min \left(\frac{1}{16\left\|M^{(q)}\right\|_{B M O_{1}(P)}}, \frac{1}{4 j\left\|M^{(q)}\right\|_{B M O_{2}(P)}^{2}}\right) .
$$

Since the $B M O_{1}$ - and the $B M O_{2}$-norms are equivalent, the lemma will be proved if

$$
\sup _{q \leq q_{0}}\left\|M^{(q)}\right\|_{B M O_{2}(P)}<\infty .
$$

To show this, we recall that the $Z^{(q)}$ satisfy $R_{\log L}(P)$ and condition $(S)$ uniformly in $q$. Claim (4.9) then follows by Lemma 2.2.

It follows from Lemmas 4.11 and 4.12 that

$$
\left(f_{p}\right)_{p \geq r+1} \text { is bounded in } L^{r}(P) \text { for every } r \geq p_{0} .
$$

LEMMA 4.13. Under our standing assumptions, for every sequence $\left(p_{n}\right)_{n}$ with $p_{n} \rightarrow \infty$ there exists a measurable $f$ such that

$$
\lim _{p_{n} \rightarrow \infty} f_{p_{n}}=f \quad \text { in probability. }
$$

PROOF. We argue by contradiction: Suppose that $\left(f_{p_{n}}\right)_{n}$ does not converge in probability. Then there exists $\delta>0$ such that for all $M>0$ there exists a subsequence, still denoted by $\left(f_{p_{n}}\right)_{n}$, such that, with $r_{n m}:=f_{p_{n}}-f_{p_{m}}$, we have

$$
P\left(\left|r_{n m}\right|>\delta\right)>\delta
$$

for all $n, m>M$. According to (4.10), we have

$$
J:=\sup _{p_{m} \geq 3}\left\|f_{p_{m}}\right\|_{L^{2}(P)}<\infty .
$$

By Chebyshev's inequality, together with (4.13), we can find $K \equiv K(\delta, J)$ large enough that

$$
P\left(e^{f_{p_{m}}} \leq \frac{1}{K}\right)=P\left(-f_{p_{m}} \geq \log K\right)<\frac{\delta}{2} .
$$


Combining (4.12) and (4.14), we find that

$$
P\left(e^{f_{p_{m}}}\left|r_{n m}\right|>\frac{\delta}{K}\right) \geq P\left(e^{f_{p_{m}}}>\frac{1}{K},\left|r_{n m}\right|>\delta\right)>\frac{\delta}{2} .
$$

Therefore, setting $\gamma:=\frac{1}{2} \min \left(\frac{\delta}{K}, \frac{\delta}{2}\right)$, we get

$$
P\left(e^{f_{p m}}\left|r_{n m}\right|>2 \gamma\right)>2 \gamma .
$$

Since $\left(f_{p_{n}}\right)_{n}$ is uniformly integrable by (4.13), it follows that

$$
\lim _{L \rightarrow \infty} P\left(\left|f_{p_{n}}\right|>L\right)=0
$$

uniformly in $n$. Define

$$
\Gamma \equiv \Gamma(n, m, L):=\left\{\left|f_{p_{n}}\right| \leq L\right\} \cap\left\{\left|f_{p_{m}}\right| \leq L\right\} .
$$

We can find by (4.15) and (4.16) an $L$ sufficiently large that we have for all $n, m$ large enough

$$
\begin{gathered}
P(\Gamma)>1-\gamma, \\
P\left(\mathbf{1}_{\Gamma} e^{f_{p m}}\left|r_{n m}\right|>\gamma\right)>\gamma .
\end{gathered}
$$

Consider now

$$
g_{n m}:=\left(1+\frac{f_{p_{n}}}{p_{n}-1}\right)^{p_{n}-1} r_{n m}=C_{p_{n}}^{-1} Z_{T}^{\left(q_{n}\right)} r_{n m} .
$$

As by Lemmas 4.8, 4.10 and 2.2,

$$
\left(Z_{T}^{\left(q_{n}\right)}\right)_{n} \text { is bounded in } L^{\mu}(P) \text { for a } \mu>1,
$$

$\left(C_{p_{n}}^{-1}\right)_{n}$ is bounded by (4.8) and $\left(f_{p_{n}}\right)_{p_{n} \geq r+1}$ is bounded in $L^{r}(P)$ for every $r \geq p_{0}$ by (4.10), we conclude that $\left(g_{n m}\right)_{n, m}$ is uniformly integrable. Furthermore, the $g_{n m}$ have zero expectation for $n, m$ large enough because, for all $n, Q^{\left(q_{n}\right)} \in \mathcal{M}_{\mu}^{e}(P)$ by (4.19) and, with $\mu^{\prime}$ conjugate to $\mu, r_{n m}=f_{p_{n}}-f_{p_{m}}$ is in $K_{\mu^{\prime}}$ for $n, m$ large enough.

Hence we can find for every $\varepsilon>0$ a possibly even larger $L \equiv L(\varepsilon)$ such that (4.17) and (4.18) are still true and we have for all $n, m$ large enough

$$
\begin{aligned}
\left|\int_{\Gamma} g_{n m} d P\right| & =\left|\int g_{n m} d P-\int_{\Gamma^{c}} g_{n m} d P\right| \\
& \leq \int_{\Gamma^{c}}\left|g_{n m}\right| d P \\
& <\frac{\varepsilon}{2} .
\end{aligned}
$$

Since

$$
\left(1+\frac{x}{n-1}\right)^{n-1} \rightarrow e^{x}
$$


uniformly on $[-L,+L]$, it follows from the definition of $g_{n m}$ and (4.20) that there exists $M \equiv M(\varepsilon, L)$ such that for all $n, m>M$ we have additionally to (4.12) that

$$
\begin{aligned}
& \left|\int_{\Gamma} e^{f_{p_{n}}} r_{n m} d P\right|<\varepsilon, \\
& \left|\int_{\Gamma} e^{f_{p_{m}}} r_{n m} d P\right|<\varepsilon ;
\end{aligned}
$$

hence

$$
\left|\int_{\Gamma} r_{n m} e^{f_{p_{m}}}\left(1-e^{r_{n m}}\right) d P\right|<2 \varepsilon .
$$

Moreover we get for all $m>M$, using (4.21) and (4.8),

$$
\begin{gathered}
\left|E\left[\mathbf{1}_{\Gamma}\left(\left(1+\frac{f_{p_{m}}}{p_{m}-1}\right)^{p_{m}-1}-e^{f_{p_{m}}}\right)\right]\right|<1, \\
E\left[\mathbf{1}_{\Gamma}\left(1+\frac{f_{p_{m}}}{p_{m}-1}\right)^{p_{m}-1}\right] \leq E\left[\left(1+\frac{f_{p_{m}}}{p_{m}-1}\right)^{p_{m}-1}\right] \leq 1 ;
\end{gathered}
$$

hence

$$
\tau(n, m):=E\left[\mathbf{1}_{\Gamma} e^{f_{p m}}\right]<2 .
$$

Moreover, for every given $\varepsilon, \tau(n, m)$ is uniformly bounded away from zero by (4.17) and the definition of $\Gamma$. Therefore, we will henceforth just write $\tau$, suppressing the dependence on $n, m$. Let us now fix $m>M$ and define a probability measure $Q \equiv Q(n, m)$ by

$$
\frac{d Q}{d P}=\frac{\mathbf{1}_{\Gamma} e^{f_{p_{m}}}}{\tau} .
$$

By (4.18), we get

$$
\begin{aligned}
\tau \int\left|r_{n m}\right| d Q & =\int \mathbf{1}_{\Gamma} e^{f_{p_{m}}}\left|r_{n m}\right| d P \\
& >\gamma P\left(\mathbf{1}_{\Gamma} e^{f_{p_{m}}}\left|r_{n m}\right|>\gamma\right) \\
& >\gamma^{2}
\end{aligned}
$$

for all $n$ large enough. As by the definition of $\Gamma$, the $\left(r_{n m}\right)_{n}$ are bounded in $L^{1}(Q)$ by $2 L$, we thus may find a finite $\eta^{\prime} \geq \gamma^{2}>0$ such that, after further extracting a subsequence still denoted by $\left(r_{n m}\right)_{n}$, we have

$$
\tau \int\left|r_{n m}\right| d Q \rightarrow \eta^{\prime}
$$


Hence, for all $n$ large enough,

$$
\tau \int\left|r_{n m}\right| d Q \in[\eta, \eta+\varepsilon]
$$

where $\eta=\gamma^{2} \vee\left(\eta^{\prime}-\varepsilon / 2\right)$. On the other hand, by (4.23),

$$
\tau\left|\int r_{n m} d Q\right|<\varepsilon
$$

We now closely follow the argument in the proof of Grandits (1999), Lemma 3.4.

For $n$ large enough, we have (with $r \equiv r_{n m}$ )

$$
\begin{aligned}
& \tau \int|r| d Q \in[\eta, \eta+\varepsilon], \\
& \tau\left|\int r d Q\right|<\varepsilon .
\end{aligned}
$$

By writing $r^{+}=\frac{1}{2}(|r|+r), r^{-}=\frac{1}{2}(|r|-r)$ we get

$$
\begin{aligned}
& \tau \int r^{+} d Q \in\left[\frac{\eta-\varepsilon}{2}, \frac{\eta}{2}+\varepsilon\right], \\
& \tau \int r^{-} d Q \in\left[\frac{\eta-\varepsilon}{2}, \frac{\eta}{2}+\varepsilon\right] .
\end{aligned}
$$

Jensen's inequality now yields

$$
\begin{aligned}
\int e^{r} r d Q & =\int e^{r^{+}} r^{+} d Q-\int e^{-r^{-}} r^{-} d Q \\
& \geq \exp \left(\int r^{+} d Q\right) \int r^{+} d Q-\int e^{-r^{-}} r^{-} d Q \\
& \geq e^{(\eta-\varepsilon) / 2 \tau}\left(\frac{\eta-\varepsilon}{2 \tau}\right)-\frac{\eta+2 \varepsilon}{2 \tau} \\
& \geq \frac{\eta^{2}}{8 \tau^{2}},
\end{aligned}
$$

where the last line follows by using $e^{x} \geq 1+x$ and $\varepsilon \ll \tau$. Hence we get, using (4.25), that

$$
\begin{aligned}
\int_{\Gamma} e^{f_{p m}+r_{n m}} r_{n m} d P & =\tau \int e^{r_{n m}} r_{n m} d Q \\
& \geq \tau \frac{\eta^{2}}{8 \tau^{2}} \\
& >\frac{\gamma^{4}}{16} .
\end{aligned}
$$


Let us write

$$
\begin{aligned}
& a=\int_{\Gamma} e^{f_{p_{m}}} r_{n m} d P, \\
& b=\int_{\Gamma} e^{f_{p_{m}}+r_{n m}} r_{n m} d P .
\end{aligned}
$$

By (4.23) and (4.26) as well as (4.24), we have with $\kappa=\frac{\gamma^{4}}{16}, \kappa$ independent of $\varepsilon$, that, for all $\varepsilon>0$,

$$
|a|<\varepsilon, \quad|b|>\kappa, \quad|a-b|<2 \varepsilon .
$$

By choosing $\varepsilon$ small enough, we get a contradiction.

COROLlARY 4.14. Under our standing assumptions, for every sequence $\left(p_{n}\right)_{n}$ with $p_{n} \rightarrow \infty$ we can extract a subsequence, still denoted by $\left(p_{n}\right)_{n}$, such that there exists

$$
f \in \bigcap_{r \geq 1} K_{r}
$$

with

$$
\lim _{p_{n} \rightarrow \infty} f_{p_{n}}=f, \quad P \text {-a.s. and in } L^{r}(P)
$$

for every $r \geq 1$.

PROOF. The statement about $P$-a.s. convergence follows from the preceding lemma. As by (4.10) the family $\left(f_{p}\right)_{p \geq r+1}$ is bounded in $L^{r}(P)$ for every $r \geq p_{0}$, the convergence holds by uniform integrability in $L^{r}(P)$ for every $r \geq p_{0}$ (and hence for every $r \geq 1$ ) as well. Finally, as $f_{p} \in K_{r}$ for all $p$ large enough and the spaces $K_{r}$ are closed in $L^{r}(P)$ by definition, we have $f \in \bigcap_{r \geq 1} K_{r}$.

Let us now state the main result of this section. We recall that $f_{p}={ }^{0} f_{p}$ and $C_{p}=C_{p, 0}$.

THEOREM 4.15. Under our standing assumptions,

$$
\lim _{q \rightarrow 1} Q^{(q)}=Q^{E} \quad \text { in entropy, }
$$

where $Q^{E}$ is the minimal entropy martingale measure.

PROOF. Since $\left(f_{p}\right)_{p}$ converges $P$-a.s., at least along a subsequence, and $\varphi_{p}(x) \uparrow e^{x}$, we get that for every pair of sequences $\left(q_{n}\right)_{n},\left(p_{n}\right)_{n}$ with $q_{n} \rightarrow 1$ and 
such that $1 / p_{n}+1 / q_{n}=1$ we can extract subsequences, still denoted by $\left(q_{n}\right)_{n}$, $\left(p_{n}\right)_{n}$, such that, with $\bar{Z}_{T}$ from Corollary 4.9 , we have

$$
\begin{aligned}
\lim _{q_{n} \rightarrow 1} Z_{T}^{\left(q_{n}\right)} & =\lim _{p_{n} \rightarrow \infty} C_{p_{n}} \varphi_{p_{n}}\left(f_{p_{n}}\right) \\
& =c \exp (f)=\bar{Z}_{T}, \quad P \text {-a.s., }
\end{aligned}
$$

where $c=\lim _{p_{n} \rightarrow \infty} C_{p_{n}}$ is, by (4.8) and (4.6), a positive finite constant and $f=\lim _{p_{n} \rightarrow \infty} f_{p_{n}}, P$-a.s.

By Lemma 4.8 , the family $\left(Z_{T}^{(q)}\right)_{q}$ is uniformly integrable; hence the convergence in (4.29) is in $L^{1}(P)$ too. Let us denote by $\bar{Q} \in \mathcal{M}(P)$ the martingale measure with density $\bar{Z}_{T}$. As the density processes $Z^{(q)}$ satisfy $R_{L \log L}(P)$ by Lemma 4.8 uniformly in $q$, we conclude by the conditional version of Fatou's lemma that the density process $\bar{Z}$ of $\bar{Q}$ satisfies $R_{L \log L}(P)$.

Now let $\bar{M}$ denote the stochastic logarithm of $\bar{Z}$, that is, $\bar{Z}=\mathscr{E}(\bar{M})$, and let $\bar{E}$ denote expectation with respect to $\bar{Q}$. As by Doob's maximal inequality the convergence in (4.29) actually is uniform in probability, it follows from Lemma 4.10 that $\bar{Z}$ satisfies condition $(S)$. We then get from Lemma 2.2 that $\bar{M} \in B M O(P)$ and that $\bar{Z}$ satisfies the reverse Hölder inequality $R_{1+\varepsilon}(P)$ for some $\varepsilon>0$; hence

$$
\frac{d \bar{Q}}{d P} \in L^{1+\varepsilon}(P) \quad \text { for some } \varepsilon>0 .
$$

Moreover, by Proposition 5 of Doléans-Dade and Meyer (1979), $\bar{Z}$ satisfies the Muckenhoupt condition $A_{s}(P)$ for some $s>1$. It follows that

$$
\frac{d P}{d \bar{Q}} \in L^{\varepsilon}(P) \quad \text { for some } \varepsilon>0 \text {. }
$$

From (4.28) and (4.30), we get by Hölder's inequality that

$$
\lim _{p_{n} \rightarrow \infty} f_{p_{n}}=f \quad \text { in } L^{1}(\bar{Q}) \text {. }
$$

As $f_{p_{n}}$ is for $p_{n}$ large enough in the $L^{1}(\bar{Q})$-closure of $\mathcal{V}$ by (4.30), this is true for $f$ too. By Remark III.2 of Stricker (1990) we conclude that

$$
f=\left(\int \eta d X\right)_{T}
$$

for an $X$-integrable $\eta$ such that $\int \eta d X$ is a uniformly integrable $\bar{Q}$-martingale. We want to show next that $\int \eta d X \in B M O(\bar{Q})$. As [compare (2.3) for the notation $\widehat{\bar{M}}$ ]

$$
c^{-1} \exp \left(-\left(\int \eta d X\right)_{T}\right)=\bar{Z}_{T}^{-1}=\mathcal{E}(\widehat{\bar{M}})_{T},
$$

we get by the formula for the stochastic exponential

$$
-\left(\int \eta d X\right)_{t}=\log c+\bar{E}\left[\widehat{\bar{M}}_{T}-\frac{1}{2}\left[\widehat{M}_{T}^{c}+U_{T} \mid \mathcal{F}_{t}\right],\right.
$$


where

$$
U_{t}=\sum_{0 \leq s \leq t}\left\{\log \left(1+\Delta \widehat{\bar{M}}_{s}\right)-\Delta \widehat{\bar{M}}_{s}\right\}
$$

As $\bar{M} \in B M O(P)$, by Proposition 6 of Doléans-Dade and Meyer (1979), $\widehat{\bar{M}} \in$ $B M O(\bar{Q})$. Therefore, we get for each stopping time $S \leq T$ that

$$
\bar{E}\left[\left|\int_{S}^{T} \eta_{t} d X_{t}\right| \mid \mathcal{F}_{S}\right] \leq \mathrm{I}+\mathrm{II}+\mathrm{III},
$$

where

$$
\begin{aligned}
\mathrm{I} & =\bar{E}\left[\left|\widehat{\bar{M}}_{T}-\widehat{\bar{M}}_{S}\right| \mid \mathcal{F}_{S}\right] \\
& \leq \bar{E}\left[\left|\widehat{\bar{M}}_{T}-\widehat{\bar{M}}_{S-}\right| \mid \mathcal{F}_{S}\right]+\left|\Delta \widehat{\bar{M}}_{S}\right| \leq C
\end{aligned}
$$

uniformly since $\widehat{\bar{M}}$ is a $B M O(\bar{Q})$-martingale (and has therefore uniformly bounded jumps);

$$
\begin{aligned}
\mathrm{II} & =\frac{1}{2} \bar{E}\left[\left|[\widehat{\bar{M}}]_{T}^{c}-\bar{E}\left[[\widehat{\bar{M}}]_{T}^{c} \mid \mathcal{F}_{S}\right]\right| \mid \mathcal{F}_{S}\right] \\
& =\frac{1}{2} \bar{E}\left[\left|[\widehat{\bar{M}}]_{T}^{c}-\bar{E}\left[[\widehat{\bar{M}}]_{T}^{c}-[\widehat{\bar{M}}]_{S}^{c} \mid \mathcal{F}_{S}\right]-[\widehat{\bar{M}}]_{S}^{c}\right| \mid \mathcal{F}_{S}\right] \\
& \leq \bar{E}\left[[\widehat{\bar{M}}]_{T}^{c}-[\widehat{\bar{M}}]_{S}^{c} \mid \mathcal{F}_{S}\right] \leq C
\end{aligned}
$$

uniformly since $\widehat{\bar{M}}$, and hence $\widehat{\bar{M}}^{c}$ too, is a $B M O(\bar{Q})$-martingale;

$$
\begin{aligned}
\mathrm{III} & =\bar{E}\left[\left|U_{T}-\bar{E}\left[U_{T} \mid \mathcal{F}_{S}\right]\right| \mid \mathcal{F}_{S}\right] \\
& \leq 2 \bar{E}\left[\sum_{S \leq t \leq T}\left\{\Delta \widehat{\bar{M}}_{t}-\log \left(1+\Delta \widehat{\bar{M}}_{t}\right)\right\} \mid \mathcal{F}_{S}\right] \\
& \leq 2 J \bar{E}\left[\sum_{S \leq t \leq T}\left(\Delta \widehat{\bar{M}}_{t}\right)^{2} \mid \mathcal{F}_{S}\right] \\
& \leq 2 J \bar{E}\left[[\widehat{\widehat{M}}]_{T}^{d}-\left[\widehat{\bar{M}}_{S}^{d} \mid \mathcal{F}_{S}\right]\right. \\
& \leq C
\end{aligned}
$$

uniformly since $\widehat{\bar{M}}$, and hence $\widehat{\bar{M}}^{d}$ too, is a $B M O(\bar{Q})$-martingale. Note that for the second inequality we have used condition $(S)$ for $\widehat{M}$ as in (2.2). Summing up, we have shown that

$$
\sup _{S \leq T}\left\|\bar{E}\left[\left|\int_{S}^{T} \eta_{t} d X_{t}\right| \mid \mathcal{F}_{S}\right]\right\|_{L^{\infty}(P)}<\infty
$$


It follows by the continuity of $X$ that

$$
\int \eta d X \in B M O(\bar{Q}) \text {. }
$$

$\bar{Q}$ has finite relative entropy with respect to $P$ since its density process satisfies $R_{L \log L}(P)$. Hence we conclude by (4.31), (4.32), (4.33) and Proposition 3.4 that $\bar{Q}$ is the minimal entropy martingale measure. We shall now show that the $Q^{\left(q_{n}\right)}$ converge to $\bar{Q}$ in entropy: we have, since $f \in \bigcap_{r} K_{r}$ by (4.27), that $E\left[Z_{T}^{\left(q_{n}\right)} f\right]=0$. Therefore,

$$
\begin{aligned}
I\left(Q^{\left(q_{n}\right)}, \bar{Q}\right) & =E\left[Z_{T}^{\left(q_{n}\right)} \log Z_{T}^{\left(q_{n}\right)}\right]-E\left[Z_{T}^{\left(q_{n}\right)} \log \left(\bar{Z}_{T}\right)\right] \\
& =\log C_{p_{n}}+C_{p_{n}} E\left[\varphi_{p_{n}}\left(f_{p_{n}}\right) \log \varphi_{p_{n}}\left(f_{p_{n}}\right)\right]-\log c .
\end{aligned}
$$

Since $-1 / e \leq x \log x$ for $x>0$ and $\log \varphi_{p}(x) \leq x$ for $x>-(p-1)$, we get

$$
\begin{aligned}
-\frac{1}{e} & \leq E\left[\varphi_{p_{n}}\left(f_{p_{n}}\right) \log \varphi_{p_{n}}\left(f_{p_{n}}\right)\right] \\
& \leq E\left[\varphi_{p_{n}}\left(f_{p_{n}}\right) f_{p_{n}}\right] \\
& =0 .
\end{aligned}
$$

The nonnegativity of the relative entropy together with $C_{p_{n}} \rightarrow c$ now implies that actually

$$
\lim _{p_{n} \rightarrow \infty} E\left[\varphi_{p_{n}}\left(f_{p_{n}}\right) \log \varphi_{p_{n}}\left(f_{p_{n}}\right)\right]=0
$$

hence

$$
\lim _{q_{n} \rightarrow 1} I\left(Q^{\left(q_{n}\right)}, \bar{Q}\right)=0 .
$$

Finally, as the minimal entropy martingale measure is unique, every subsequence $\left(Q^{\left(q_{n}\right)}\right)_{n}$ has the same limit $\bar{Q}$ and we end up with

$$
\lim _{q \rightarrow 1} I\left(Q^{(q)}, \bar{Q}\right)=0 .
$$

EXAMPLE. We consider in this example a class of diffusion models where we have an additional random factor influencing the coefficients of the diffusion, which is independent of the driving Brownian motion. By a conditioning argument, we derive explicit formulae for the various optimal martingale measures studied in this paper.

Let $\Omega=C[0, T] \times D[0, T]$. For $\omega=\left(\omega_{0}, y\right)$ let $W_{t}(\omega)=\omega_{0}(t)$ and $Y_{t}(\omega)=$ $y(t)$ denote the coordinate processes. We set $\mathbb{F}=\mathbb{F}^{W} \times \mathbb{F}^{Y}$ and $P=P_{W} \otimes P_{Y}$, where $P_{W}$ is the Wiener measure and $P_{Y}$ denotes the distribution of $Y$. Moreover, for each $y \in D[0, T]$ we denote by $P_{y}$ the regular conditional probability given $Y=y$. 
Consider then the stochastic differential equation

$$
d X_{t}=\mu\left(t, Y_{.}\right) X_{t} d t+\sigma\left(t, Y_{.}\right) X_{t} d W_{t},
$$

where $\mu, \sigma: \mathbb{R}_{+} \times D[0, T] \rightarrow \mathbb{R}$ are such that for each $y \in D[0, T]$ the processes $\mu(t, y(t))$ and $\sigma(t, y(t))$ are adapted and have left continuous paths with right limits. Moreover, we assume that

$$
\begin{aligned}
|\mu(t, y)| \leq C(y) & \forall(t, y) \in \mathbb{R}_{+} \times D[0, T], \\
0<c(y) \leq|\sigma(t, y)| \leq C(y) \quad \forall(t, y) & \in \mathbb{R}_{+} \times D[0, T],
\end{aligned}
$$

where $c(y), C(y)$ are positive finite constants. Hence a strong solution of (4.34) exists; in fact, it is given by

$$
X_{t}=\mathscr{E}\left(\int \sigma d W+\int \mu d s\right)_{t}
$$

Here $Y$ is thought to model an additional random source which is independent of the driving Brownian motion $W$.

Let $M=\int \sigma X d W$ and $\lambda=\frac{\mu}{\sigma^{2} X}$ so that we get $X=M+\int \lambda d[M]$. Let us set $y=\mathcal{F}_{T}^{Y}$, the $\sigma$-algebra containing all information about $Y$. We have that

$$
K=\int \lambda^{2} d[M]=\int \frac{\mu^{2}}{\sigma^{2}} d t \quad \text { is } y \text {-measurable, }
$$

and this allows us to derive explicit formulae for various optimal martingale measures under the assumption that

$$
\int \lambda d M=\int \frac{\mu}{\sigma} d W \in B M O(P) .
$$

This is, for example, satisfied if $K=\left[\int \lambda d M\right]$ is uniformly bounded, which is a common assumption in applications to mathematical finance. By Theorem 2.3 of Kazamaki (1994) and Girsanov's theorem,

$$
Z_{T}:=\mathscr{E}\left(-\int \lambda d M\right)_{T}
$$

is the density of an equivalent martingale measure and the density process $Z$ satisfies, by Doléans-Dade and Meyer [(1979), Proposition 6], a reverse Hölder inequality $R_{q_{0}}(P)$ for a $q_{0}>1$. Moreover,

$$
\text { the spaces } G_{T}^{p} \text { are closed in } L^{p}(P) \text { for } p \geq p_{0}, 1 / p_{0}+1 / q_{0}=1 \text {, }
$$

by Theorem 4.1 of Grandits and Krawczyk (1998). Furthermore, we can infer from Theorem 3.1 of Grandits and Krawczyk (1998) that $\Theta^{p}=L^{p}(M)$. We get, 
with $r=1 /(p-1)$,

$$
\begin{aligned}
Z^{r} & =\mathscr{E}\left(-\int \lambda d M\right)^{r} \\
& =\mathcal{E}\left(-\int \lambda d X\right)^{r} \exp (r K) \\
& =\&\left(-r \int \lambda d X\right) \exp \left(\frac{1}{2}(1+r) r K\right) ;
\end{aligned}
$$

hence

$$
\begin{aligned}
\exp \left(-\frac{1}{2}\left(1+\frac{1}{p-1}\right) K\right) Z & =\varepsilon\left(-\frac{1}{p-1} \int \lambda d X\right)^{p-1} \\
& =\left(1+\int \vartheta_{p} d X\right)^{p-1}
\end{aligned}
$$

for some $X$-integrable $\vartheta_{p}$. We are now going to show that, for $p \geq p_{0}$,

$$
\vartheta_{p} \in \Theta^{p} \text {. }
$$

By the weighted Burkholder-Davis-Gundy inequality [see Choulli, Krawczyk and Stricker (1997) for a recent treatment], the fact that $p-1=p / q$ together with the estimate

$$
\exp \left(-\frac{q}{2}\left(1+\frac{1}{p-1}\right) K_{T}\right) \leq 1
$$

and the (ordinary) Doob inequality, it follows for $p \geq p_{0}$ that

$$
\begin{aligned}
E\left[\left(\int \vartheta_{p}^{2} d[M]\right)_{T}^{p / 2}\right] & =E\left[\left(\int \vartheta_{p}^{2} d[X]\right)_{T}^{p / 2}\right] \\
& \leq C E\left[\left(\left(\int \vartheta_{p} d X\right)_{T}^{*}\right)^{p}\right] \\
& \leq C\left(E\left[\left(Z^{*}\right)_{T}^{q}\right]+1\right) \\
& \leq C\left(E\left[Z_{T}^{q}\right]+1\right) \\
& <\infty \quad \text { for } q \leq q_{0} ;
\end{aligned}
$$

hence $\vartheta_{p} \in L^{p}(M)=\Theta^{p}$. Now let, for $q \leq q_{0}$,

$$
\bar{Z}_{T}^{(q)}=C_{p} \exp \left(-\frac{1}{2}\left(1+\frac{1}{p-1}\right) K_{T}\right) Z_{T},
$$

where $C_{p}$ is a normalizing constant such that $E\left[\bar{Z}_{T}^{(q)}\right]=1$. Let us now show that $\bar{Z}_{T}^{(q)}$ gives the density of a martingale measure; by (4.37), (4.38) and 
Proposition 4.2 we then conclude that $\bar{Z}_{T}^{(q)}$ is the density $Z_{T}^{(q)}$ of the $q$-optimal martingale measure. The idea is to make use of the independence between $W$ and $Y$ by conditioning on $y$. Let us first collect some facts.

For $A \in \mathcal{F}_{T}$ and $f \in \mathcal{L}^{0}\left(\Omega, \mathcal{F}_{T}, P\right)$ we set

$$
\begin{aligned}
A_{y} & =\left\{\omega_{0} \mid\left(\omega_{0}, y\right) \in A\right\}, \\
f^{y}\left(\omega_{0}\right) & =f\left(\omega_{0}, y\right) .
\end{aligned}
$$

Furthermore, for a process $U$ we define $U^{y}$ via $\left(U^{y}\right)_{t}=\left(U_{t}\right)^{y}$.

REMARK (i). Let $S$ be an $\mathbb{F}$-stopping time. Then $S^{y}$ is an $\mathbb{F}^{W}$-stopping time for every $y$.

Proof. As $\{S \leq t\} \in \mathcal{F}_{t}, t \in[0, T]$, we get by Fubini $\left\{S^{y} \leq t\right\}=\{S \leq t\}_{y} \in$ $\mathcal{F}_{t}^{W}$.

REMARK (ii). If $S$ is an $\mathbb{F}$-stopping time and $h \in \mathcal{L}^{\infty}\left(\mathcal{F}_{S}, P\right)$, then $h^{y} \in$ $\mathcal{L}^{\infty}\left(\mathcal{F}_{S^{y}}^{W}, P_{y}\right)$ for every $y$.

PRoOF. For every $A \in \mathscr{F}_{S}$ and every $t \in[0, T]$ we have $A \cap\{S \leq t\} \in \mathcal{F}_{t}$ and therefore $A_{y} \cap\left\{S^{y} \leq t\right\}=(A \cap\{S \leq t\})_{y} \in \mathcal{F}_{t}^{W}$. Therefore the statement is true for indicator functions, and a monotone class argument yields the result.

REMARK (iii). If $t \rightarrow \vartheta(t, y)$ is left continuous with right limits for each $y$, then

$$
\left(\int \vartheta d W\right)^{y}=\int \vartheta^{y} d W
$$

Proof. The statement follows by (i) and (ii) for elementary stochastic integrals with respect to $W$. So it remains to approximate $\left(\int \vartheta d W\right)_{t}, 0 \leq t \leq T$, in probability by elementary stochastic integrals and to use the fact that $f_{n} \rightarrow f$ in probability implies $f_{n}^{y} \rightarrow f^{y}$ in probability by passing over to almost surely convergent subsequences.

Now since both $Z$ and $X$ can be written as stochastic exponentials of $-\int \frac{\mu}{\sigma} d W$ and $\int \mu d t+\int \sigma d W$, respectively, we get from (iii) that $Z_{T}^{y}$ is the density of a martingale measure for the solution of (4.34) with $Y=y$. Therefore $E\left[Z_{T}^{y} f^{y}\right]=0$ for each $y$ and each $f \in \mathcal{V}$; hence, by independence of $W$ and $Y$,

$$
E\left[Z_{T} f \mid \mathcal{y}\right]=0 \quad \text { for each } f \in \mathcal{V} .
$$

Therefore, as $K$ is $\mathcal{y}$-measurable, we get, for all $f \in \mathcal{V}$,

$$
E\left[\bar{Z}_{T}^{(q)} f\right]=E\left[\exp \left(-\frac{1}{2}\left(1+\frac{1}{p-1}\right) K_{T}\right) E\left[Z_{T} f \mid y\right]\right]=0 ;
$$


hence $\bar{Z}_{T}^{(q)}$ is indeed the density $Z_{T}^{(q)}$ of the $q$-optimal martingale measure.

It follows by monotone convergence that

$$
\lim _{q \rightarrow 1} C_{p}^{-1} Z_{T}^{(q)}=\exp \left(-\frac{1}{2} K_{T}\right) Z_{T}=\exp \left(-\left(\int \lambda d X\right)_{T}\right) \quad \text { in } L^{1}(P) .
$$

Hence, with

$$
c=E\left[\exp \left(-\left(\int \lambda d X\right)_{T}\right)\right]^{-1},
$$

we get from (4.36), Theorem 4.15 and the fact that convergence in entropy implies $L^{1}$-convergence of the densities that $c \exp \left(-\left(\int \lambda d X\right)_{T}\right)$ is the density of the minimal entropy martingale measure. Note that this expression is in general different from the density $\mathcal{E}\left(-\int \lambda d M\right)_{T}$ of the minimal martingale measure $\widehat{P}$ which minimizes the reverse relative entropy $I(P, Q)$ over all equivalent martingale measures $Q$; compare Schweizer (1999a).

\section{APPENDIX}

The following considerations are in principle well known but are not stated elsewhere explicitly in this form.

We work in the setting as stated just before Lemma 2.2. Note that if $Z$ satisfies condition $(S)$ with constant $s$, then there exist $\delta, C>0$ such that

$$
-1+\delta \leq \Delta M \leq C
$$

(one might choose $\delta=1 / s, C=s-1$ ) and vice versa. To be consistent with the notation in Kazamaki (1979), in this Appendix $C$ does not denote a generic constant. We henceforth assume that $M$ (resp. $\widehat{M}$ ) satisfies (A.1) and that $Z, \widehat{Z}$ satisfy condition $(S)$ with constant $s$. In Doléans-Dade and Meyer [(1979), Propositions 5, 6] it was proved that then the following assertions are equivalent:

(i) $\widehat{M} \in B M O(Q)$;

(ii) $\widehat{Z}$ satisfies $A_{r}(Q)$ for some $r>1$;

(iii) $\widehat{Z}$ satisfies $R_{p}(Q)$ for some $p>1$;

(iv) $Z$ satisfies $A_{q}(P)$ for some $q>1$;

(v) $Z$ satisfies $R_{r^{\prime}}(P)$ for some $r^{\prime}>1$;

(vi) $M \in B M O(P)$.

Let $\|\widehat{M}\|_{B M O_{2}(Q)}<L$. It was also proved, but not stated, that one can choose the corresponding constants in the implications (i) $\Rightarrow$ (vi) in such a way that they only depend on $L$ and $s$. By symmetry, an analogous statement is then true for the other direction as well. Readers are asked to go to the proofs in Doléans-Dade and Meyer (1979) and Kazamaki $(1979,1994)$ for themselves, as we only give a guideline how to verify this claim. 
(i) $\Rightarrow$ (ii). Here we follow the proof of the theorem in Kazamaki (1979). He introduces $m \equiv m(\delta)$ such that $m>\max (1 / \delta, 2)$ and defines for an $\alpha \in(0,1)$ certain functions $x_{\alpha}, y_{\alpha}$ which only depend on $m$ and $\alpha$; moreover one has $x_{\alpha} \rightarrow-1+\frac{1}{m}$ and $y_{\alpha} \rightarrow \infty$ as $\alpha \rightarrow 0$. We modify Kazamaki's proof slightly by choosing $\alpha \in(0,1)$ such that $x_{\alpha}<-1+\delta, y_{\alpha} \geq L$ and $\alpha<2 /\left(m L^{2}\right)$; this is possible by the foregoing and moreover it implies Kazamaki's requirement for $\alpha$. He then shows that $\&\left(\widehat{M}^{d}\right)$ satisfies $A_{r_{1}}(Q)$ for $r_{1}=1+2 / \alpha$ where one can take as constant $\left(1-m \alpha L^{2} / 2\right)^{-1 / 2}$. Moreover, as $\left\|\widehat{M}^{c}\right\|_{B M O_{2}(Q)} \leq\|\widehat{M}\|_{B M O_{2}(Q)}$, it follows from Kazamaki [(1994), proof of Theorem 2.4, (a) $\Rightarrow$ (b)] that if $r_{2}$ is large enough that $L<\sqrt{2}\left(\sqrt{r_{2}}-1\right)$, then $\mathscr{E}\left(\widehat{M}^{c}\right)$ satisfies $A_{r_{2}}(Q)$ with constant $2^{\left(\sqrt{r_{2}}+1\right) / \sqrt{r_{2}}}$. As $\mathcal{E}(\widehat{M})=\mathscr{E}\left(\widehat{M}^{c}\right) \mathcal{E}\left(\widehat{M}^{d}\right)$ we conclude by Hölder's inequality that $\mathscr{E}(\widehat{M})$ satisfies $A_{r}(Q)$ for some $r>1$ large enough with some constant which depends only on $\delta$ (hence $s$ ) and $L$.

(ii) $\Rightarrow$ (iii). Let $\mathscr{E}(\widehat{M})$ satisfy $A_{r}(Q)$ with some constant which we write as $K^{1 /(r-1)}$. We now follow the arguments in the proof of Lemma 3 in Kazamaki (1979). He introduces $a=2^{r} K, b_{\varepsilon, K}=2(1+C) \varepsilon a^{1+\varepsilon} /(1+\varepsilon)$ and chooses $\varepsilon>0$ such that $b_{\varepsilon, K}<1$. Then it is shown that $\mathcal{E}(\widehat{M})$ satisfies $R^{1+\varepsilon}(Q)$ with constant $C_{\varepsilon, K}=\left(3-b_{\varepsilon, K}\right) /\left(1-b_{\varepsilon, K}\right)$; hence $C_{\varepsilon, K}$ depends only on $\delta, C$ and $L$.

(iii) $\Rightarrow$ (iv). Obvious.

(iv) $\Rightarrow$ (vi). It is shown in Doléans-Dade and Meyer [(1979), Proposition 6A] that if $Z$ satisfies $A_{q}(P)$ for some $q>1$ with constant $K$ [and condition $(S)$ ] then $\|M\|_{B M O_{2}(P)} \leq \sqrt{(q-1) / j \cdot 2 \log K}+C$, where $j$ is a positive constant less than or equal to $1 / 2$ which depends only on $s$.

(iv) $\Rightarrow$ (v). The proof is analogous to (ii) $\Rightarrow$ (iii).

Therefore, the claim was indeed implicitly proved in the 1970s.

\section{REFERENCES}

Choulli, T., Krawczyk, L. and Stricker, C. (1997). On Fefferman and Burkholder-DavisGundy inequalities for $\mathcal{E}$-martingales. Probab. Theory Related Fields 113 571-597.

Choulli, T., KRAWCZYK, L. and STRICKER, C. (1998). E-martingales and their applications in mathematical finance. Ann. Probab. 26 853-876.

CSISzÁr, I. (1975). I-divergence geometry of probability distributions. Ann. Probab. 3 146-158.

Delbaen, F., Grandits, P., Rheinländer, T., SAmperi, D., Schweizer, M. and STRICKER, C. (2000). Exponential hedging and entropic penalties. Math. Finance 12 99-123.

Delbaen, F., Monat, P., Schachermayer, W., Schweizer, M. and Stricker, C. (1997). Weighted norm inequalities and hedging in incomplete markets. Finance and Stochastics 1 181-228.

Delbaen, F. and Schachermayer, W. (1996a). Attainable claims with pth moments. Ann. Inst. H. Poincaré Math. Statist. 32 743-763.

Delbaen, F. and Schachermayer, W. (1996b). The variance-optimal martingale measure for continuous processes. Bernoulli 2 81-105.

Dellacherie, C. and Meyer, P.-A. (1982). Probabilities and Potential B. North-Holland, Amsterdam. 
DoléAns-Dade, C. and Meyer, P. A. (1979). Inégalités de normes avec poids. Séminaire de Probabilités XIII. Lecture Notes in Math. 721 313-331. Springer, Berlin.

FRITTELLI, M. (2000). The minimal entropy martingale measure and the valuation problem in incomplete markets. Math. Finance 10 39-52.

Grandits, P. (1999). The p-optimal martingale measure and its asymptotic relation with the minimal entropy martingale measure. Bernoulli 5 225-247.

Grandits, P. and KRAWCZYK, L. (1998). Closedness of some spaces of stochastic integrals. Séminaire de Probabilités XXXII. Lecture Notes in Math. 1686 73-85. Springer, Berlin.

JACOD, J. (1979). Calcul stochastique et problèmes de martingales. Lecture Notes in Math. 714. Springer, Berlin.

Jeulin, T. and Yor, M. (1979). Sur l'expression de la dualité entre $\mathscr{H}^{1}$ et BMO. Séminaire de Probabilités XIII. Lecture Notes in Math. 721 360-370. Springer, Berlin.

KAZAMAKI, N. (1979). A sufficient condition for the uniform integrability of exponential martingales. Math. Rep. Toyama Univ. 2 1-11.

KaZAmaki, N. (1994). Continuous Exponential Martingales and BMO. Lecture Notes in Math. 1579. Springer, Berlin.

KRAWCZYK, L. (1997). Hedging derivative securities in incomplete markets. Ph.D. dissertation, Warsaw Univ.

Luenberger, D. (1969). Optimization by Vector Space Methods. Wiley, New York.

Revuz, D. and Yor, M. (1990). Continuous Martingales and Brownian Motion. Springer, Berlin.

RHEINLÄNDER, T. and SCHWEIZER, M. (1997). On $L^{2}$-projections on a space of stochastic integrals. Ann. Probab. 25 1810-1831.

SCHWEIZER, M. (1994). Approximating random variables by stochastic integrals. Ann. Probab. 22 $1536-1575$.

SCHWEIZER, M. (1996). Approximation pricing and the variance-optimal martingale measure. Ann. Probab. 24 206-236.

SCHWEIZER, M. (1999a). A minimality property of the minimal martingale measure. Statist. Probab. Lett. 42 27-31.

SCHWEIZER, M. (1999b). A guided tour through quadratic hedging approaches. Preprint, Technische Univ. Berlin.

Stricker, C. (1990). Arbitrage et lois de martingale. Ann. Inst. H. Poincaré 26 451-460.

Yor, M. (1978). Sous-espaces denses dans $L^{1}$ ou $H^{1}$ et représentation des martingales. Séminaire de Probabilités XII. Lecture Notes in Math. 649 265-309. Springer, Berlin.

STATISTICAL LABORATORY

CAMBRIDGE UNIVERSITY

16 Mill Lane

CAMBRIDGE CB2 $1 \mathrm{SB}$

UNITED KINGDOM
DePartment of MATHEMATics

ETH-ZENTRUM

HG G51.2

CH-8092 ZÜRICH

SWITZERLAND

E-MAIL: rhein@math.ethz.ch 